\title{
Speciation dynamics during the global radiation of extant bats
}

\author{
Jeff J. Shi ${ }^{1,2}$ and Daniel L. Rabosky ${ }^{1}$ \\ ${ }^{1}$ Department of Ecology and Evolutionary Biology and Museum of Zoology, University of Michigan, Ann Arbor,
} Michigan 48109

${ }^{2}$ E-mail: jeffjshi@umich.edu

Received December 18, 2014

Accepted May 4, 2015

\begin{abstract}
Species richness varies widely across extant clades, but the causes of this variation remain poorly understood. We investigate the role of diversification rate heterogeneity in shaping patterns of diversity across families of extant bats. To provide a robust framework for macroevolutionary inference, we assemble a time-calibrated, species-level phylogeny using a supermatrix of mitochondrial and nuclear sequence data. We analyze the phylogeny using a Bayesian method for modeling complex evolutionary dynamics. Surprisingly, we find that variation in family richness can largely be explained without invoking heterogeneous diversification dynamics. We document only a single well-supported shift in diversification dynamics across bats, occurring at the base of the subfamily Stenodermatinae. Bat diversity is phylogenetically imbalanced, but-contrary to previous hypotheses-this pattern is unexplained by any simple patterns of diversification rate heterogeneity. This discordance may indicate that diversification dynamics are more complex than can be captured using the statistical tools available for modeling data at this scale. We infer that bats as a whole are almost entirely united into one macroevolutionary cohort, with decelerating speciation through time. There is also a significant relationship between clade age and richness, suggesting that global bat diversity may still be expanding.
\end{abstract}

KEY WORDS: BAMM, Chiroptera, diversification rates, macroevolution, phylogenetic imbalance.

One of the most striking trends across the tree of life is the unequal distribution of diversity across extant clades (Raup et al. 1973; Gould et al. 1987; McPeek and Brown 2007; Alfaro et al. 2009). Although many groups of organisms have dramatically radiated over evolutionary timescales, others have stagnated or failed to diversify, and remain species-poor. Evolutionary extremes at both ends of the spectrum, across the tree of life, have long captivated evolutionary biologists and ecologists. For example, angiosperms and beetles are famously species-rich, whereas groups ranging from tuataras to coelacanths have persisted at low diversity (Farrell 1998; Magallón and Sanderson 2001; Harmon 2012). What has driven this pervasive pattern of unequal species richness throughout the tree of life?

A long-standing hypothesis is that unequal diversity stems from diversification rate variation across clades (Stanley 1979; Strathmann and Slatkin 1983; Kirkpatrick and Slatkin 1993; Barraclough and Nee 2001; Chan and Moore 2002; Wiens 2011). Proposed factors that control diversification rate heterogeneity invoke both biotic interactions among organisms as well as the external influences of environment and geography (Vrba 1992; Barnosky 2001; Benton 2009; Badgley and Finarelli 2013). Diversification rates may vary based on ecological and geographic opportunity, as in the presence or absence of competitors, depopulated niche spaces, or the opening of niche space following the evolution of a key innovation (Simpson 1953; Benton 1987; Erwin et al. 1987; Rosenzweig and McCord 1991; Schluter 2000; Yoder et al. 2010; Rabosky 2013). Quantifying diversification rate variation is the first step to potentially uncovering ecological and geographical drivers of extant diversity patterns.

In this study, we investigate macroevolutionary patterns across extant bats (order Chiroptera). Bats are the second-most species-rich order of extant mammals and are distributed across nearly all terrestrial biomes (Nowak 1994; Simmons 2005b). Their taxonomic diversity is complemented by considerable morphological and ecological diversity. Bat trophic ecology includes insectivory, vertebrate carnivory, frugivory, nectarivory, 
and sanguivory (Nowak 1994; Simmons and Conway 2003). Bat diversity is also not equally distributed across the order: some clades of bats have radiated into a variety of species, ecological niches, and biogeographic zones, while others remain restricted or conserved (Jones et al. 2005; Simmons 2005b). For example, New World leaf-nosed bats are known for both high species richness and morphological disparity, whereas the closely related New Zealand short-tailed and bulldog bats are species-poor with highly specialized morphologies.

The more than 1300 species of extant bats are partitioned into 20 ecologically and geographically heterogeneous families (Simmons 2005b). Six families of extant bats are more species-rich than all others: Pteropodidae, Rhinolophidae, Hipposideridae, Phyllostomidae, Molossidae, and Vespertilionidae encompass roughly $75 \%$ of extant species diversity (Fig. 1). This apparent imbalance of diversity may be driven by clade-specific differences in diversification rate (Jones et al. 2005). For example, the Neotropical phyllostomids are thought to have shifted to higher diversification rates in association with their ecological diversification (Monteiro and Nogueira 2011; Dumont et al. 2012; Santana et al. 2012). The evolution of the leaf-nose in both phyllostomids and the Old World rhinolophoids has been considered a key innovation that precipitated rapid diversification and high species richness (Teeling et al. 2002; Fenton 2010). Other researchers have inferred accelerated diversification in the cosmopolitan vespertilionids, perhaps due to increased substitution rates or heightened transposon activity (Lack and Van Den Bussche 2010; Platt et al. 2014). Frugivory in both phyllostomids and pteropodids has been considered a key innovation that may have driven accelerated diversification within both families (Almeida et al. 2011; Rojas et al. 2012). Overall, many potential macroevolutionary processes can be evaluated in this highly diverse clade of animals.

Although it is possible that unequal bat clade diversity is driven by diversification rate shifts, it is also possible that imbalance reflects clade ages. The radiation of crown Chiroptera likely dates to the early Cenozoic, as with other major mammalian lineages (Simmons 2005a; Simmons et al. 2008; Meredith et al. 2011; Raia et al. 2012). Identifying significant diversification rate shifts is only possible with accurate time calibration. However, phylogenetic resolution and time-calibration across the order have historically been difficult, given the notoriously poor bat fossil record and numerous systematic revisions (Eiting and Gunnell 2009; Teeling et al. 2012). Although the backbone of bat families is well-resolved (Teeling et al. 2005), a robust timecalibrated, species-level phylogeny encompassing the majority of extant bat diversity has remained elusive. Recent efforts have focused on higher levels, such as genus, or are limited to one or few genetic loci (Agnarsson et al. 2011; Yu et al. 2014).
We present an analysis of macroevolutionary dynamics across extant bats. We first assembled a time-calibrated, specieslevel phylogeny of bats by aggregating multilocus genetic data and incorporating fossil dating across extant families. We then quantified diversification rates to test whether the variation in species richness across bats (Fig. 1) is a product of diversification rate heterogeneity, or whether it largely reflects other factors, such as the amount of time available for diversity to accumulate.

\section{Methods SEQUENCE ALIGNMENT}

We mined GenBank for all available bat mitochondrial and nuclear sequences, utilizing scripts that automated sequence identification, cleaning, and alignment, and generally followed the supermatrix approaches of Hinchcliff and Roalson (2013) and Zanne et al. (2014). We first used the PhyLoTa Browser (Sanderson et al. 2008) to identify all loci sequenced for at least 20 unique bat species within GenBank release 194. We then downloaded the entire national center for biotechnology information (NCBI) SQLite3 database of Chiroptera nucleotide data using the program PHLAWD (Smith et al. 2009). Within this set, we identified "guide sequences" for each of the aforementioned candidate loci from PhyLoTa. Guide sequences aided in identification of homologous loci, and were selected to maximize family-level coverage. We chose the longest sequence for each of the monotypic bat families. For all other families, we chose either the two genera with the longest sequences, or for the highly diverse families Vespertilionidae and Phyllostomidae, the four genera with the longest sequences. Based on these guide sequences, we used PHLAWD to parse the NCBI database and identify sequences for each locus. We plotted basic local alignment search tool (BLAST) scores of coverage against identity for these sequences, and excluded outliers. Finally, we assembled alignments for each locus, with one representative sequence per taxon. We also included sequences for three mammalian outgroups: Canis lupus and Sorex araneus from the same superorder (Laurasiatheria), and Mus musculus as an even earlier diverging outgroup.

Each alignment was cleaned in the program Gblocks (Castresana 2000; Talavera and Castresana 2007), which identified conserved regions while allowing at most $50 \%$ gaps at any site. Finally, we concatenated all loci into a supermatrix with the program Phyutility (Smith and Dunn 2008). Each locus was specified as an independent partition for all subsequent phylogenetic analyses. Using the taxonomy of Simmons (2005b), we collapsed all subspecies to the longest species-level sequence, and removed ambiguous species. For genera described since 2005, we collapsed to species-level sequences using the NCBI taxonomy. 


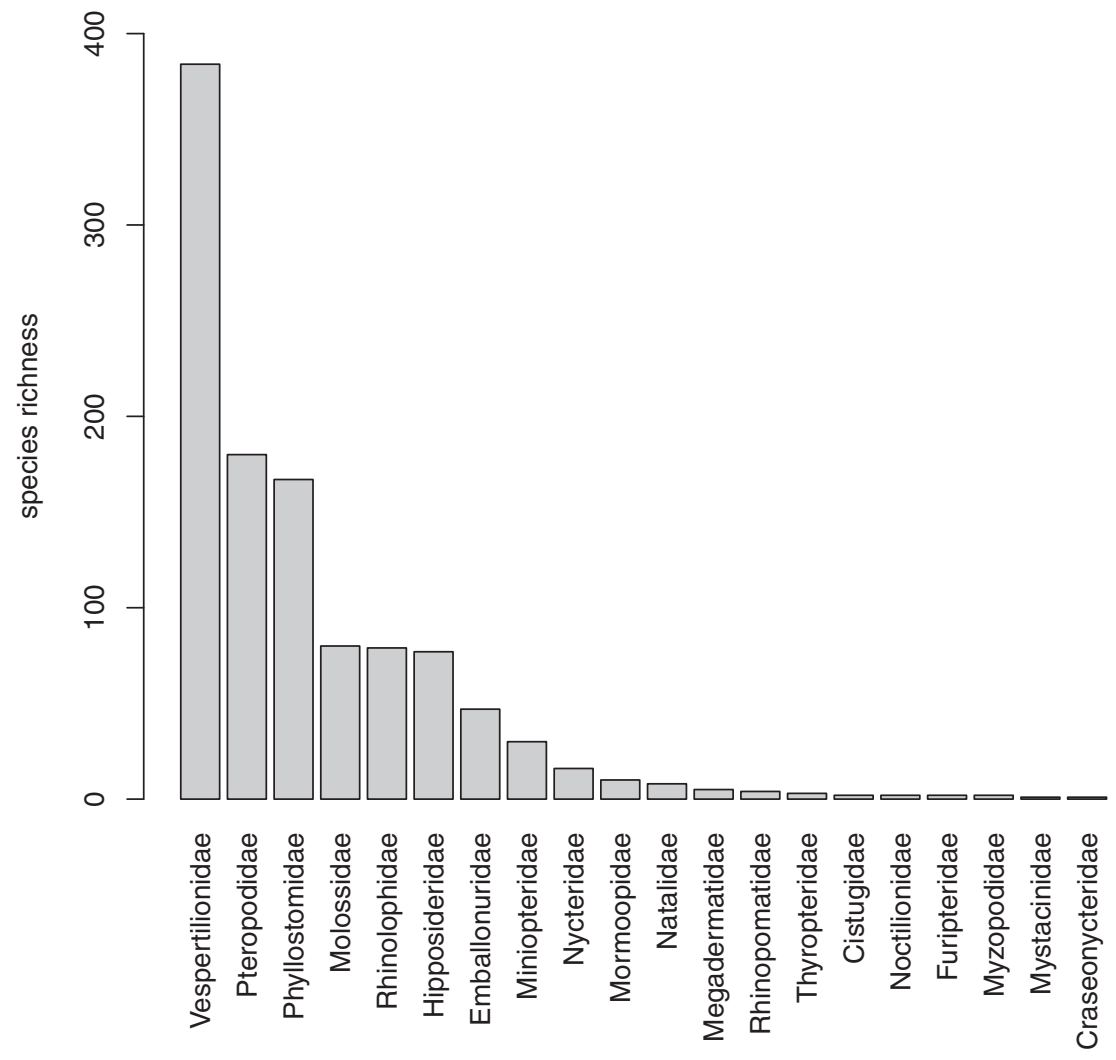

Figure 1. Species richnesses of all extant bat families in rank-order, taken from Simmons (2005b).

Our final alignment included all 20 extant families. The seven families represented by the most individual loci were the six largest families-Vespertilionidae, Phyllostomidae, Pteropodidae, Molossidae, Rhinolophidae, Hipposideridae—and Nycteridae (Fig. 1), which together comprised $87 \%$ of species in our dataset. The concatenated and cleaned dataset included 29 loci, and totaled 20,376 bp (Table 1). All alignments and PHLAWD output, with Genbank annotations for included sequences, have been archived in the Dryad repository.

\section{PHYLOGENY CONSTRUCTION}

We estimated the maximum-likelihood (ML) phylogeny and bootstrap support using RAxML version 8 (Stamatakis 2014). We parameterized RAxML by assigning a separate GTR $+\Gamma$ model of rate heterogeneity to each locus, and initiated runs with maximum parsimony trees. To improve likelihood calculation, we constrained topologies to an established backbone, as in other large-scale phylogenies (e.g., Zanne et al. 2014). This backbone included the Yangochiroptera and Yinpterochiroptera suborders, and within those, the Rhinolophoidea, Emballonuroidea, Noctilionoidea, and Vespertilionoidea superfamilies of Teeling et al. (2005). For calibration purposes, we additionally enforced the monophyly of the large vespertilionid subfamily Myotinae.
After an initial ML search, we used the program RogueNaRok to identify "rogue taxa" (Aberer et al. 2013). Rogue taxa can bias phylogenetic inference, with low phylogenetic signal or even sequence misidentification within GenBank (Hinchliff and Roalson 2013). We also pruned two extremely long branch lengths to avoid biases of long-branch attraction. We performed one final ML tree search after pruning (see Table S1), and also 100 rapid bootstrap analyses to assess support.

Using fossils, we time-calibrated the ML phylogeny using penalized likelihood as implemented in the program treePL (Sanderson 2002; Smith and O'Meara 2012). treePL explicitly allowed for rate variation across branches, but penalized rate differences after cross-validating initial analyses. We used 24 fossil calibration points (Table 2) as described by Jones et al. (2002, 2005), Teeling et al. (2003, 2005), and/or were described and validated in the Paleobiology Database (PaleobDB). We followed PaleobDB taxonomy for consistency. An upper bound was also set for the divergence between crown Chiroptera and carnivores at the K-Pg boundary (65.5 million years ago [mya]; though see Springer et al. 2003; Teeling et al. 2003; Bininda-Emonds et al. 2007; Meredith et al. 2011).

Our fossils generally defined minimum dates for crown families, subfamilies, and genera, and were placed upon our ML phylogeny as such in treePL. We tested the correlation of our 
Table 1. Loci included in this study, as well as relevant information on location, length, phylogenetic coverage, and the GenBank coverage/identity scores used to parameterize PHLAWD searches and alignments.

\begin{tabular}{|c|c|c|c|c|c|c|c|}
\hline Locus & Abbreviation & Genome & $\begin{array}{l}\text { Base } \\
\text { Pairs }\end{array}$ & Taxa & Families & $\begin{array}{l}\text { Coverage } \\
\text { Cutoff }\end{array}$ & $\begin{array}{l}\text { Identity } \\
\text { Cutoff }\end{array}$ \\
\hline $\begin{array}{l}\text { 12S, tRNA-valine, and/or } \\
\quad 16 \mathrm{~S}\end{array}$ & 12S-tRNAVal-16S & Mitochondrion & 2306 & 269 & 15 & 0 & 0.62 \\
\hline Adenosine A3 receptor & ADORA3 & Nucleus & 320 & 33 & 7 & 0 & 0 \\
\hline Beta- 2 adrenergic receptor & ADRB2 & Nucleus & 700 & 32 & 7 & 0 & 0 \\
\hline Apolipoprotein B & apoB & Nucleus & 277 & 135 & 7 & 0 & 0.5 \\
\hline Amyloid precursor protein & APP & Nucleus & 612 & 28 & 7 & 0.2 & 0.2 \\
\hline $\begin{array}{l}\text { Copper-transporting } \\
\text { ATPase } 1\end{array}$ & ATP7A & Nucleus & 628 & 35 & 7 & 0 & 0 \\
\hline $\begin{array}{l}\text { Brain-derived neurotrophic } \\
\text { factor }\end{array}$ & BDNF & Nucleus & 533 & 33 & 7 & 0 & 0 \\
\hline Beta fibrinogen & BFIB & Nucleus & 624 & 57 & 7 & 0.4 & 0.2 \\
\hline $\begin{array}{l}\text { Breast cancer type } 1 \\
\text { susceptibility protein }\end{array}$ & BRCA1 & Nucleus & 1316 & 85 & 7 & 0.2 & 0.5 \\
\hline $\begin{array}{l}\text { c-mos oocyte maturation } \\
\text { protooncogene }\end{array}$ & C-MOS & Nucleus & 463 & 41 & 7 & 0.8 & 0.85 \\
\hline $\begin{array}{l}\text { Cytochrome oxidase } \\
\text { subunit } 1\end{array}$ & $\mathrm{COI}$ & Mitochondrion & 651 & 489 & 16 & 0.2 & 0.2 \\
\hline Cytochrome b & CYTB & Mitochondrion & 706 & 743 & 18 & 0 & 0 \\
\hline $\begin{array}{l}\text { Endothelial differentiation } \\
\text { gene } 1\end{array}$ & EDG1 & Nucleus & 370 & 30 & 7 & 0 & 0 \\
\hline $\begin{array}{l}\text { Potassium voltage-gated } \\
\text { channel subfamily KQT } \\
\text { member } 4\end{array}$ & KCNQ4 & Nucleus & 160 & 22 & 6 & 0.2 & 0 \\
\hline $\begin{array}{l}\text { Reduced nicotinamide } \\
\text { adenine dinucleotide } \\
\text { (NADH) dehydrogenase } \\
\text { subunit } 1\end{array}$ & ND1 & Mitochondrion & 798 & 236 & 11 & 0.2 & 0.2 \\
\hline $\begin{array}{l}\text { NADH dehydrogenase } \\
\text { subunit } 2\end{array}$ & ND2 & Mitochondrion & 1043 & 163 & 7 & 0.22 & 0.22 \\
\hline Blue-sensitive opsin & OPN1SW & Nucleus & 1895 & 35 & 7 & 0.6 & 0.6 \\
\hline Prepronociceptin & PNOC & Nucleus & 269 & 32 & 7 & 0 & 0 \\
\hline Protein kinase C Iota & PRKC1 & Nucleus & 369 & 174 & 10 & 0.5 & 0.625 \\
\hline $\begin{array}{l}\text { Recombination activating } \\
\text { protein } 1\end{array}$ & RAG1 & Nucleus & 768 & 129 & 7 & 0.75 & 0.8 \\
\hline $\begin{array}{l}\text { Recombination activating } \\
\text { protein } 2\end{array}$ & RAG2 & Nucleus & 752 & 335 & 15 & 0.7 & 0.7 \\
\hline $\begin{array}{l}\text { Spectrin nonerythroid beta } \\
\text { chain } 1\end{array}$ & SPTBN & Nucleus & 574 & 44 & 7 & 0.4 & 0.4 \\
\hline $\begin{array}{l}\text { Signal transducer and } \\
\text { activator of transcription } \\
5 \mathrm{~A}\end{array}$ & STAT5A & Nucleus & 461 & 146 & 7 & 0.2 & 0.2 \\
\hline $\begin{array}{l}\text { Taste receptor type } 1 \\
\text { member } 2\end{array}$ & TAS1R2 & Nucleus & 711 & 42 & 7 & 0 & 0 \\
\hline $\begin{array}{l}\text { Thyroid stimulating } \\
\text { hormone beta }\end{array}$ & TSHB & Nucleus & 407 & 109 & 7 & 0.625 & 0.6 \\
\hline Titin & TTN & Nucleus & 1155 & 33 & 7 & 0.4 & 0.3 \\
\hline Tyrosinase & TYR & Nucleus & 325 & 31 & 7 & 0.2 & 0.2 \\
\hline von Willenbrand factor & VWF & Nucleus & 1005 & 132 & 7 & 0 & 0 \\
\hline Zinc finger protein $X$-linked & ZFX & Nucleus & 178 & 25 & 6 & 0.3 & 0.3 \\
\hline
\end{tabular}


Table 2. Fossil taxa used to time-calibrate the phylogenies in this study, along with relevant citations and defined clades.

\begin{tabular}{|c|c|c|c|}
\hline \multicolumn{2}{|l|}{ Fossil } & \multicolumn{2}{|c|}{ Approximate Fossil } \\
\hline Constraint & Clade & Date (mya) & Citation(s) in Supporting Text \\
\hline Onychonycteris finneyi* & Chiroptera & 55.8 & Simmons et al. (2008) \\
\hline Rhinolophoidea* & Rhinolophoidea & 55 (maximum) & Teeling et al. (2005) \\
\hline Rhinolophus* & Crown Rhinolophus & 37.2 & $\begin{array}{l}\text { Crochet et al. (1981), Teeling et al. } \\
\text { (2005) }\end{array}$ \\
\hline Hipposideros* & $\begin{array}{l}\text { Crown Hipposideros, } \\
\text { internal Hipposideridae }\end{array}$ & 40.4 & Crochet et al. (1981), Sigé (1988) \\
\hline $\begin{array}{l}\text { Rhinolophidae, } \\
\text { Hipposideridae split }\end{array}$ & $\begin{array}{l}\text { Rhinolophidae, } \\
\text { Hipposideridae } \\
\text { divergence time }\end{array}$ & 37 & $\begin{array}{l}\text { Teeling et al. (2003), Almeida et al. } \\
\text { (2009) }\end{array}$ \\
\hline Macroderma* & $\begin{array}{l}\text { Crown Macroderma, } \\
\text { internal Megadermatidae }\end{array}$ & 23.03 & Hand and Archer (2005) \\
\hline $\begin{array}{l}\text { Megadermatidae, } \\
\text { Rhinopomatidae split }\end{array}$ & $\begin{array}{l}\text { Rhinopomatidae divergence } \\
\text { time }\end{array}$ & 34 & $\begin{array}{l}\text { McKenna and Bell (1997), Teeling } \\
\text { et al. (2003, 2005), Almeida et al. } \\
\text { (2009) }\end{array}$ \\
\hline Tachypteron* & Emballonuridae & 44 & Storch et al. (2002) \\
\hline Diclidurus & $\begin{array}{l}\text { Crown Diclidurus, internal } \\
\text { Emballonuridae }\end{array}$ & 13 & $\begin{array}{l}\text { Carlini et al. (1997), Czaplewski } \\
\text { (1997) }\end{array}$ \\
\hline Nycteris* & Nycteridae & 5.332 & Black and Krishtalka (1986) \\
\hline Mystacinidae & Mystacinidae & 20.1 & Hand et al. (2013) \\
\hline Thyroptera lavali* & Thyropteridae & 13 & $\begin{array}{l}\text { Czaplewski (1997), Czaplewski et al. } \\
\text { (2003) }\end{array}$ \\
\hline Mormoopidae* & Mormoopidae & 30 & $\begin{array}{l}\text { Morgan and Czaplewski (2003), } \\
\text { Teeling et al. (2005) }\end{array}$ \\
\hline Noctilio albiventris* & Noctilionidae & 13 & $\begin{array}{l}\text { Czaplewski (1997), Czaplewski et al. } \\
\text { (2003) }\end{array}$ \\
\hline Phyllostomidae* & Phyllostomidae & 34 (maximum) & Teeling et al. (2005) \\
\hline Palynephyllum* & $\begin{array}{l}\text { Crown Lonchophyllinae, } \\
\text { internal Phyllostomidae }\end{array}$ & 13 & Dávalos et al. (2014) \\
\hline $\begin{array}{l}\text { Desmodus } \\
\quad \text { archaeodaptes }\end{array}$ & $\begin{array}{l}\text { Crown Desmodontinae, } \\
\text { internal Phyllostomidae }\end{array}$ & 4.9 & Morgan (1991) \\
\hline Nataloidea* & Natalidae & 43 & $\begin{array}{l}\text { McKenna and Bell (1997), but see } \\
\text { Simmons and Geisler (1998); } \\
\text { Morgan and Czaplewski (2003) }\end{array}$ \\
\hline Molossidae* & Molossidae & 37.2 & $\begin{array}{l}\text { Arroyo-Cabrales et al. (2002), } \\
\text { Teeling et al. (2005) }\end{array}$ \\
\hline Eumops & $\begin{array}{l}\text { Crown Eumops, internal } \\
\text { Molossidae }\end{array}$ & 13 & Czaplewski et al. (2003) \\
\hline Miniopterus fossilis* & Miniopteridae & 13.65 & Sabol and Holec (2002) \\
\hline Stehlinia* & Vespertilionidae & 42.7 & $\begin{array}{l}\text { Gunnell and Simmons (2012), } \\
\text { Gunnell (pers. comm.) }\end{array}$ \\
\hline Myotis* & $\begin{array}{l}\text { Crown Myotinae, internal } \\
\text { Vespertilionidae }\end{array}$ & 27 & $\begin{array}{l}\text { Gunnell and Simmons (2012), } \\
\text { Gunnell (pers. comm.) }\end{array}$ \\
\hline
\end{tabular}

Unless otherwise indicated, dates were considered minimum constraints for the specified group(s). Constraints marked with an asterisk were also used to time calibrate our bootstrap replicates, as they were either included in the backbone during tree searching or were always recovered as monophyletic. Full citation information can be found in the Supporting Information.

inferred ML family ages with the phylogenies of Jones et al. (2005) and Teeling et al. (2005) to compare our results with previous publications. Because crown ages are likely not normally distributed, we performed pairwise Spearman's rank correlation tests for these comparisons. For these correlations, the families
Cistugidae and Miniopteridae were nested within the closely related Vespertilionidae, as was the case in Jones et al. (2005) and Teeling et al. (2005). The placement of Miniopteridae, which was elevated to family based on molecular data (Miller-Butterworth et al. 2007), has implications for bat systematics that should be 
kept in mind when considering these previous publications. We did not perform crown age tests on monotypic families.

\section{MACROEVOLUTIONARY MODELING}

We modeled macroevolutionary dynamics of diversification across bat phylogenies with the program BAMM version 2.0 (Bayesian Analysis of Macroevolutionary Mixtures: Rabosky et al. 2013; Rabosky 2014; latest version available from http:// bamm-project.org/). We used BAMM to quantify diversification rates throughout the bat clade, and to identify different macroevolutionary regimes. In the BAMM framework, regimes refer to a shared, potentially dynamic diversification process shared by all lineages downstream from the location of a rate shift. The posterior probability of a particular configuration of shifts can be estimated by the frequency that it is sampled during the analysis. The number of shifts in any given shift configuration is one more than the number of regimes, given a starting "background" regime at the root.

For our ML phylogeny, we ran 10 million generations of reversible-jump Markov chain Monte Carlo (MCMC) sampling, with samples drawn from the posterior every 1000 generations. BAMM version 2.0 (released June 2014) implements Metropoliscoupled MCMC (MCMCMC; MC3) to improve the efficiency of simulating the posterior probability distribution (Altekar et al. 2004). MC3 is a variant of MCMC sampling where multiple chains are run simultaneously. Inference about the posterior distribution is based on a single chain - the so-called "cold chain"while the remaining chains (heated chains) are used to more thoroughly explore parameter space. Heating a chain involves flattening the posterior probability distribution such that the chain is more free to wander through parameter space. Relative to the cold chain, heated chains are more likely to accept proposed states that move the chain into regions of lower posterior probability. As a consequence, heated chains are less likely to become stuck on local optima than are cold chains. The implementation of MC3 in BAMM version 2.0 follows the algorithm for chain swaps described in Altekar et al. (2004) and follows an incremental heating scheme.

To flatten a posterior probability distribution, chains are heated by modifying the acceptance probabilities of proposed states. Letting $f(\theta, M)$ denote the posterior probability of the current model $(M)$ and parameters $(\theta)$, the probability of accepting a new state with model $M^{\prime}$ ' and parameters $\theta^{\prime}$ is given by:

$$
\min \left[1,\left(\frac{f\left(\theta^{\prime}, M^{\prime}\right)}{f(\theta, M)}\right)^{\beta} Q\right],
$$

where $\beta$ is the "heat" that is applied to the chain and $Q$ is the ratio of transition probabilities between current and proposed states. If $\beta=1$, this equation reduces to standard MCMC. However, if $\beta<1$, the algorithm will accept proportionately more proposals, thus enabling the chain to wander around a seemingly flattened probability landscape. The cold chain alone is used to approximate the true posterior distribution, but MC3 periodically proposes state swaps between the cold chain and the heated chains, which improves the overall efficiency of the algorithm (Altekar et al. 2004). In BAMM, two randomly chosen chains $j$ and $k$ with respective heating parameters $\beta_{j}$ and $\beta_{k}$ are selected to exchange states with a predetermined swap frequency, and the state swap is accepted with probability:

$$
\min \left[1, \frac{f\left(M_{k}, \theta_{k}\right)^{\beta_{j}}}{f\left(M_{j}, \theta_{j}\right)^{\beta_{j}}} \frac{f\left(M_{j}, \theta_{j}\right)^{\beta_{k}}}{f\left(M_{k}, \theta_{k}\right)^{\beta_{k}}}\right] .
$$

The heat parameter $\beta$ is determined by an incremental heating scheme (Altekar et al. 2004), where a set of $n$ chains, $i \in(1,2, \ldots, n)$, combined with a temperature parameter $\Delta T$, allows for the computation of the heat for the $i$ 'th chain as:

$$
\beta_{i}=\frac{1}{1+(i-1) \Delta T} .
$$

This equation reduces to $\beta=1$ (standard MCMC) when $n=1$. For our analyses of the bat phylogeny, we performed MC3 sampling with $n=8$ chains, $\Delta T=0.1$, and swaps between chains were proposed every 1000 generations.

We estimated speciation and extinction priors with the $\mathrm{R}$ package BAMMtools version 2.0 (Rabosky et al. 2014b), and specified a value of 1.0 for the exponential hyperprior governing the number of distinct rate shift regimes. This hyperprior was chosen to be conservative and to minimize type I errors (Rabosky 2014). We ran all analyses on the University of Michigan's Flux high performance computing cluster.

We also accounted for incomplete sampling of bat diversity, which can bias inferences of diversification rates (Nee et al. 1994; Pybus and Harvey 2000; Heath et al. 2008; Rabosky and Lovette 2008), by estimating the sampling percentage of each bat genus as described by Simmons (2005b) (Table S2). BAMM incorporates analytical corrections for incomplete taxon sampling (FitzJohn et al. 2009), under the assumption of random taxon sampling at some level of taxonomic hierarchy. In our case, we assumed that species were randomly sampled within genera, which allowed us to apply separate genus sampling fractions. For those genera with higher current species counts than are described in Simmons (2005b), we specified complete sampling. If estimated diversities of bat clades are inaccurate, due to crypsis or taxonomic artifacts, diversification results may change alongside sampling fractions. To test sensitivity to incorrect estimates of clade sizes and incomplete sampling, we ran an additional analysis where we assumed that the true genus-level diversity was twice that of current estimates. Practically, this entailed running an additional analysis with all sampling fractions halved.

To investigate the effect of topological uncertainty on our macroevolutionary inferences, we also ran BAMM on the 
100 bootstrap replicate phylogenies from RAxML. We first timecalibrated all bootstrap replicates using a subset of our fossil constraints that were either topologically constrained in the tree search or were monophyletic across all replicates (Table 2). We ran 10 million generations of MCMC sampling for each individual time-calibrated bootstrap phylogeny, with replicate-specific priors on speciation and extinction estimated using BAMMtools. All other priors and parameters were identical to our ML analysis.

We checked for convergence of the MCMC algorithm by plotting likelihood scores against sampled generations, ensuring adequate mixing of the chains, and checking for effective sample sizes above at least $10 \%$ of our sampled generations. To be conservative, we then discarded the first $20 \%$ of samples as burn-in.

\section{BRANCH-SPECIFIC DIVERSIFICATION RATE SHIFTS}

BAMM enables researchers to estimate the marginal probability of a rate shift along a single branch of a phylogeny (Rabosky 2014; Rabosky et al. 2014a). The marginal probability of a rate shift is simply the frequency with which a rate shift is observed on a particular branch across the full posterior distribution of macroevolutionary rate shift configurations simulated using BAMM. Here, we develop an alternative approach that uses Bayes factors (Kass and Raftery 1995) to evaluate branch-specific evidence for a rate shift. A branch-specific Bayes factor is a measure of the evidence for a rate shift along a particular branch relative to the evidence for an alternative model without a rate shift on the branch, and takes branch length into account. Generally, the Bayes factor associated with two models $M_{j}$ and $M_{k}$ can be computed as:

$$
\frac{\frac{\operatorname{Pr}\left(M_{j}\right)}{\pi\left(M_{j}\right)}}{\frac{\operatorname{Pr}\left(M_{k}\right)}{\pi\left(M_{k}\right)}},
$$

where $\operatorname{Pr}\left(M_{j}\right)$ and $\operatorname{Pr}\left(M_{k}\right)$ are the posterior probabilities of models $j$ and $k$, and $\pi\left(M_{j}\right)$ and $\pi\left(M_{k}\right)$ are the prior probabilities of models $j$ and $k$.

In our case, for a given branch $x$, the marginal shift probability $P_{x}$ is an estimate of the posterior probability of a model with a shift, and $\left(1-P_{x}\right)$ is the posterior probability of no shift. BAMM assumes that diversification rate shifts occur on phylogenetic trees under a compound Poisson process and provides an estimate of the prior probability of a rate shift on each branch in the tree. Thus, if $\pi_{x}$ is the prior probability of a rate shift on branch $x$, $\left(1-\pi_{x}\right)$ is the prior probability of no shift on that branch. It is then straightforward to compute a branch-specific Bayes factor for branch $x$ as:

$$
\frac{\frac{P_{x}}{\pi_{x}}}{\frac{1-P_{x}}{1-\pi_{x}}}
$$

We refer to this as a "marginal" Bayes factor, because it is computed across the posterior distribution while ignoring the degree to which shift probabilities on specific branches may covary with one another. Shift probabilities along branches are not independent of those on other branches (Rabosky et al. 2014a) and cannot be interpreted as such; this also applies to the Bayes factors computed here. For example, adjacent branches often have strong negative covariances in shift occurrences (Rabosky et al. 2014a). Because the prior probability of a rate shift on a particular branch is a strict function of branch length, we interpret marginal Bayes factors as the evidence favoring rate shifts after controlling for branch length. For example, we expect to observe more shifts on long branches than on short branches, even if shifts are randomly distributed across the tree. We computed branch-specific Bayes factors for each branch in the bat phylogeny. These methods have been implemented in the current versions of BAMM and BAMMtools.

\section{COHORTS, AGE-RICHNESS, AND PHYLOGENETIC IMBALANCE}

We established different macroevolutionary cohorts across bats (Rabosky et al. 2014a). A "macroevolutionary cohort" is a set of taxa that share a common set of macroevolutionary rate parameters. For example, if a given sample from the posterior has zero rate shifts, it is necessarily true that all lineages are assigned to the same evolutionary process that began at the root of the tree. All lineages would thus be considered part of the same macroevolutionary cohort when we infer elevated pairwise probabilities of originating under the same diversification process. With this analysis, we could easily visualize any heterogeneity in macroevolutionary regimes across all bats, and identify the unique cohorts most likely to be decoupled from the rest of the order (Fig. S1).

Our cohort analysis was also performed across all bootstrap replicates, in case phylogenetic uncertainty affected cohort membership. We then calculated the posterior probabilities of different configurations of rate shifts across our ML phylogeny and bootstrap replicates. To also assess the effect of topological uncertainty upon rate shifts, we estimated a pooled distribution of macroevolutionary regime shifts across the 100 bootstrap replicates. It was most appropriate to refer to this distribution as a "quasi-posterior," as phylogenetic tree topologies were sampled by bootstrapping; a true posterior would sample them in proportion to their posterior probability. We computed Bayes factor evidence in favor of the model with the highest quasi-posterior probability over a model with no shifts.

We tested for a relationship between crown age and species richnesses of extant families, using both Spearman's rank correlation tests and phylogenetic generalized least squares (PGLS) with a Brownian correlation structure to correct for evolutionary 
nonindependence (Martins and Hansen 1997). We only assessed the effect of crown age on diversity, as the use of stem ages is known to strongly bias age-richness relationships (Stadler et al. 2014). We also used BAMMtools to calculate instantaneous rates of diversification across our ML phylogeny, for both our main run and the BAMM run with halved sampling fractions. To test for significance of nonconstant rates of diversification, we calculated the $\gamma$-statistic (Pybus and Harvey 2000) for our ML phylogeny and all bootstrap phylogenies using phytools (Revell 2012). We investigated the effect of incomplete sampling on the $\gamma$-statistic by simulating pure-birth phylogenies with varying levels of sampling using the R package geiger (Harmon et al. 2008).

To further explore the extent to which species richness in major bat clades can largely be explained by a single global diversification process, we quantified temporal patterns of bat phylogeny imbalance and compared them to a constant rate birthdeath process as a corresponding null expectation of imbalance. We operationally defined imbalance as the variance in species richness among descendant clades of all lineages present at some time $t$ in the phylogeny. To calculate this metric, we sampled the ML phylogeny in 100 "slices" evenly spaced from the root to the present. We then recorded the number of ancestral lineages $n$ present at each slice $t$, and the number of extant descendants from each of these $n$ lineages. We then calculated the empirical variance in $\log$ (species richness) among the $n$ descendant clades, for each of the 100 time slices across the phylogeny. Each point produced by this method corresponded to the variance among realized evolutionary outcomes for each of the $n$ ancestral lineages at each time $t$.

We then generated a null distribution of expected variances from the $n$ ancestral lineages present at each time $t$. For each slice, we randomly assigned the estimated 1300 extant species of bats into $n$ clades, then simulated our incomplete sampling by arbitrarily selecting 812 (the number of species in the ML phylogeny) of these 1300 . This procedure simulated expected clade diversities descended from $n$ ancestral lineages, if the descendant diversity of a clade is random with respect to its ancestral lineage. On average, the distribution of clade diversities at any time approximated a geometric rank-abundance curve (Nee et al. 1992; Rabosky 2009b). We replicated this simulation 100 times to calculate the variance among $\log$ (richnesses) of the $n$ simulated clades at each time slice $t$. In this framework, high empirical values imply a more imbalanced phylogeny, whereas low values imply more evenness distributed across clades. The null expectation of imbalance is the variance under a constant birth-death process, which we confirmed with a phylogeny simulated under this assumption using geiger (Harmon et al. 2008, Fig. S2). Imbalance may vary through time to reflect changes in macroevolutionary conditions, such as diversification rate shifts or differential diversity limits.

\section{Results \\ PHYLOGENY CONSTRUCTION}

Our time-calibrated phylogeny (Fig. 2) includes 812 species of bats, or about $62.5 \%$ of current diversity estimates. Overall, $62 \%$ of nodes are recovered with at least $70 \%$ bootstrap support (Fig. S3). This increases to $76 \%$ of nodes with at least $50 \%$ bootstrap support, and $82 \%$ of nodes with at least $40 \%$ bootstrap support. These percentages are robust across taxonomic groupings, with two notable exceptions. The superfamily Noctilionoidea (Teeling et al. 2005: families Myzopodidae, Furipteridae, Thyropteridae, Mystacinidae, Noctilionidae, Mormoopidae, Phyllostomidae) is extremely well-supported, with $75 \%$ of nodes supported by at least $70 \%$ of bootstrap replicates, and nearly $90 \%$ of nodes supported by at least $50 \%$ of bootstrap replicates. Conversely, the family Molossidae is the least well-supported, with only $62 \%$ of nodes supported by even $50 \%$ of bootstrap replicates. One systematic revision with relatively high support (70\% bootstrap support) is proposed here, with the superfamily Emballonuroidea (families Emballonuridae and Nycteridae) recovered sister to Noctilionoidea. As this was not part of the established systematic constraints used to parameterize our tree search (Teeling et al. 2005), we suggest it merits continued investigation.

We infer generally early-to-mid Eocene divergence times for the extant families of bats, ranging from approximately 40 mya to approximately 55 mya, with a crown Chiroptera age inferred around 58 mya. Correlation tests of our ML times with those of Jones et al. (2005) and Teeling et al. (2005) confirm that stem ages are generally comparable with previous research (Table 3). Spearman's rank correlation tests are highly significant, revealing a positive linear correlation between our phylogeny and that of Jones et al. (2005; $P=0.04$, Spearman's $\rho=0.488$ ) and a much stronger linear correlation between our phylogeny and that of Teeling et al. (2005; $P<0.001$, Spearman's $\rho=0.795)$. Our results notably suggest that the "Eocene big bang" of chiropteran diversification was even earlier than previously suggested (Simmons 2005a).

Crown ages among the three compared phylogenies vary the most for the families Natalidae, Noctilionidae, Rhinolophidae, and Myzopodidae. The family Natalidae, in particular, may be calibrated with fossils of uncertain placement within Nataloidea, and we include an alternate crown date that does not affect downstream analyses (Tables 2 and 3). If we exclude all of these crown ages, both pairwise tests are highly significant, with a strong, positive linear correlations between our ML crown ages and those of Jones et al. (2005; $P=0.025$, Spearman's $\rho=0.682$ ) and Teeling et al. (2005; $P=0.012$, Spearman's $\rho=0.745)$. Overall, these discrepancies should be taken into consideration for our findings, and as such we caution that the phylogeny we present is not meant to be a systematic revision of the order. Inferences for 


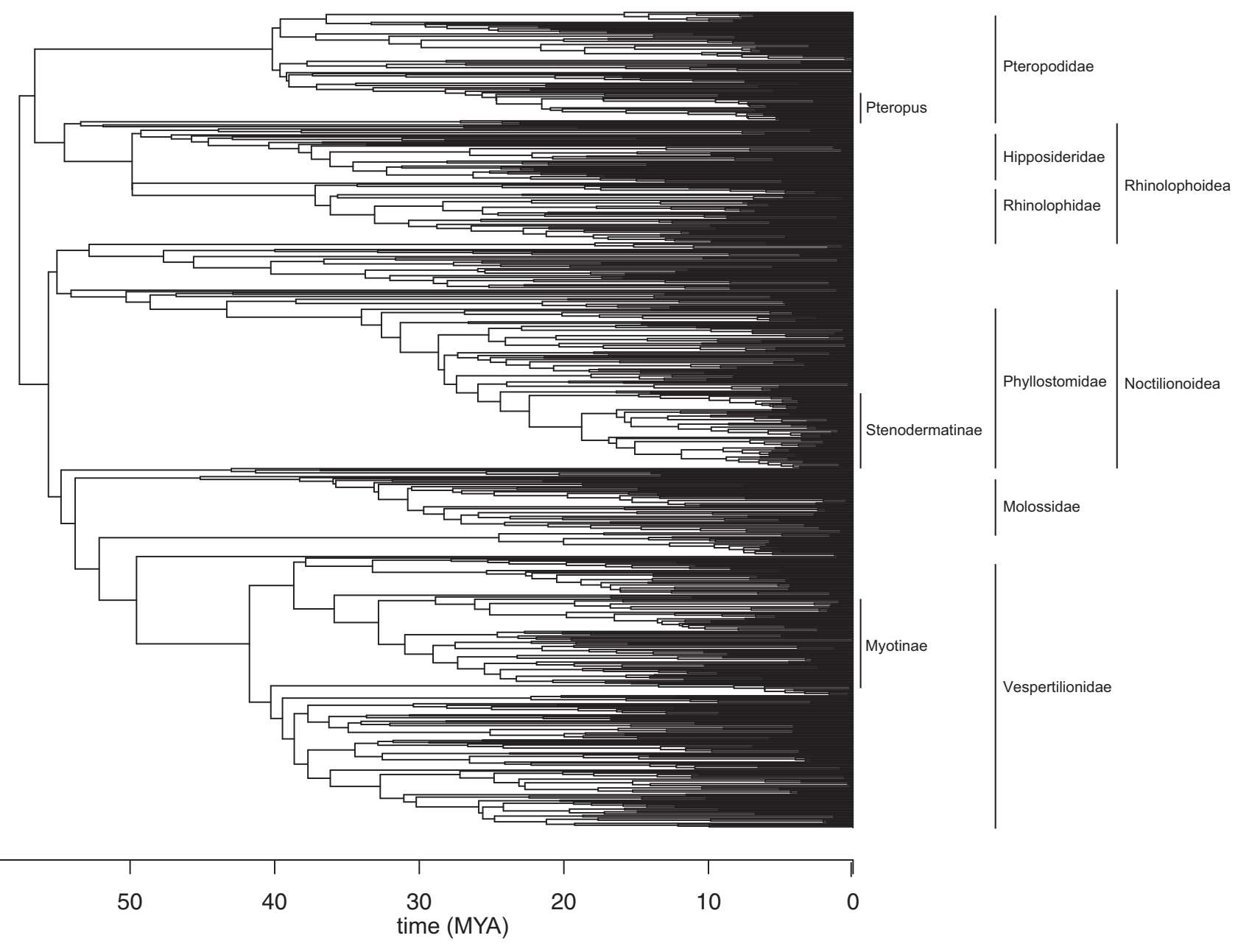

Figure 2. Time-calibrated (axis in millions of years), ML phylogeny of 812 extant species of bats. The six largest families (see Fig. 1) of bats are labeled, as well as relevant genera, subfamilies, and superfamilies for this study.

the species-rich family Rhinolophidae are the most likely to be affected, as the other families represent an extremely small percentage of extant bat diversity. Because the relative relationships among crown ages are fairly constant across our phylogeny and previous studies, we present our macroevolutionary results with the caveat that absolute dates are subject to further calibration and inference. Across our bootstrap replicates, the crown ages of extant bats vary as reported in Table 4 .

\section{MACROEVOLUTIONARY REGIMES AND RATE SHIFTS}

Although BAMM returns overall diversification rates from individual estimates of speciation and extinction rates, we report only speciation data for this study unless otherwise specified. The linear correlation between net diversification and speciation rates in our analyses is extremely positive (Pearson's $r=0.996, P<$ 0.001 ; PGLS $t=90.087, P<0.001$ ), and macroevolutionary inferences with diversification rates are highly similar, so we feel our results are robust to this consideration. Actual estimates of extinction rates are not reported considering our phylogeny is composed entirely of molecular data (Rabosky 2010).

BAMM results from our ML tree support a single rate shift and two macroevolutionary regimes, with the majority of extant bat diversity being governed by a common background regime. The post burn-in posterior distribution of the number of rate shifts is right-skewed (with a mean of 1.35 shifts and a median of 1 shift). In this distribution, a single shift occurs with a posterior probability (pp) of $70.5 \%$, and two shifts occur with $\mathrm{pp}=23.8 \%$. A configuration of zero shifts only occurs with $\mathrm{pp}=3 \%$.

We use both branch-specific Bayes factors and marginal shift probabilities to localize regime shift information on the ML phylogeny. Both metrics strongly favor a model that includes a shift at the base of Stenodermatinae over a model without a shift in this tree (Fig. 3). Importantly, it is conceptually possible for these two metrics to be in conflict, depending on the phylogeny. The compound Poisson process used in BAMM assumes a uniform probability density of rate shifts across phylogenies, leading to a 
Table 3. Comparisons of family-level stem ages/crown ages (for families with more than one representative), in millions of years, for our ML phylogeny, and for the previous studies of Jones et al. (2005) and Teeling et al. (2005).

\begin{tabular}{lcll}
\hline Family & This Study & $\begin{array}{l}\text { Jones } \\
\text { et al. (2005) }\end{array}$ & $\begin{array}{l}\text { Teeling } \\
\text { et al. }(2005)\end{array}$ \\
\hline Vespertilionidae & $52.1 / 51.1$ & $47.1 / 47.0$ & $49.3 / 49.2$ \\
Molossidae & $53.8 / 45.2$ & $47.1 / 35.7$ & $49.3 / 38.2$ \\
Natalidae & $54.8 / 43.0$ & $50.1 / 15.1$ & $51.4 / 17.3$ \\
& $(22.2)$ & & \\
Phyllostomidae & $43.3 / 34.0$ & $37.1 / 27.4$ & $38.8 / 28.1$ \\
Mormoopidae & $43.3 / 39.2$ & $37.1 / 33,7$ & $38.8 / 34.2$ \\
Thyropteridae & $46.8 / 13.8$ & $50.2 / 12.9$ & $42.1 / 15.0$ \\
Noctilionidae & $42.9 / 13.0$ & $42.7 / 3.0$ & $36.2 / 2.6$ \\
Furipteridae & $42.9 / \mathrm{NA}$ & $50.1 / 0.1$ & $36.2 / 0.1$ \\
Mystacinidae & $50.3 / \mathrm{NA}$ & $42.8 / 42.8$ & $46.1 / 46.1$ \\
Myzopodidae & $54.1 / 1.1$ & $51.8 / 51.8$ & $51.6 / 51.6$ \\
Emballonuridae & $52.8 / 47.7$ & $53.7 / 45.0$ & $52.1 / 46.1$ \\
Nycteridae & $52.8 / 17.9$ & $43.4 / 26.2$ & $52.1 / 26.1$ \\
Rhinolophidae & $49.9 / 49.8$ & $28.7 / 6.5$ & $34.9 / 8.7$ \\
& $(37.2)$ & & \\
Hipposideridae & $49.9 / 49.3$ & $28.7 / 26.5$ & $34.9 / 34.8$ \\
Rhinopomatidae & $51.9 / 26.9$ & $12.0 / 9.5$ & $39.0 / 19.4$ \\
Craseonycteridae & $51.9 / \mathrm{NA}$ & $12.0 / 12.0$ & $38.9 / 38.9$ \\
Megadermatidae & $53.4 / 27.2$ & $43.5 / 39.2$ & $38.9 / 38.9$ \\
Pteropodidae & $56.6 / 40.2$ & $61.7 / 36.1$ & $55.8 / 24.6$ \\
\hline & & & \\
\hline
\end{tabular}

For this table, Vespertilionidae includes the now-separate families of Vespertilionidae, Miniopteridae, and Cistugidae. For Natalidae and Rhinolophidae, alternate crown dates are also provided by excluding their fossils (see Table 2 and Supporting Information for Natalidae discussion), but usage of these dates do not affect any results.

Table 4. Variation in family-level (nonmonotypic) crown ages (in millions of years) across all bootstrap replicate phylogenies.

\begin{tabular}{llll}
\hline Family & Mean & Median & Range \\
\hline Vespertilionidae & 51.1 & 51.1 & $46.0-54.1$ \\
Molossidae & 40.4 & 39.4 & $37.2-46.8$ \\
Mormoopidae & 38.1 & 38.2 & $30.6-41.3$ \\
Thyropteridae & 14.1 & 14 & $13.0-16.5$ \\
Emballonuridae & 46.8 & 46.9 & $44.0-48.5$ \\
Nycteridae & 18.1 & 18.1 & $15.0-19.8$ \\
Rhinolophidae & 47.6 & 47.5 & $45.9-54.3$ \\
Hipposideridae & 46.5 & 46.6 & $40.4-51.9$ \\
Rhinopomatidae & 27 & 26.5 & $17.5-34.2$ \\
Pteropodidae & 32.6 & 30 & $22.0-41.0$ \\
\hline
\end{tabular}

For this table, Vespertilionidae includes the now-separate families of Vespertilionidae, Miniopteridae, and Cistugidae. We report the mean and median inferences, and the full range, in millions of years. Other unreported families (Natalidae, Noctilionidae, Myzopodidae, Phyllostomidae, Megadermatidae) do not vary at this precision across bootstrap replicates, and match the inferences found in Table 3. positive correlation between branch length and the prior probability of a rate shift along a branch. As such, the branch-specific Bayes factors are essentially marginal probabilities weighted by the phylogenetic branch length. Theoretically, one could observe a low marginal probability of a rate shift along a very short branch, yet-if the prior probability of a shift along the branch is sufficiently small-the Bayes factor associated with a shift on the branch may be extremely high. This would imply that the "density" of rate shifts per unit branch length is high. In the case of extant bats, we infer that the concordance between both metrics is strong evidence for a stenodermatine diversification rate shift, even after taking into account the prior distribution of rate shifts expected based on topology.

The low number of inferred shifts is not likely a product of topological uncertainty, given results from our bootstraps. Across the post burn-in BAMM results of all bootstrap replicates combined (the quasi-posterior), there is a different distribution of shifts than the prior (Fig. 4). Notably, there is extremely low quasi-posterior probability of zero shifts $(3.15 \%)$, and the highest quasi-posterior probability is for one shift. The Bayes factor evidence in favor of this model over a model with zero shifts is 120.6, indicating very strong support.

The $95 \%$ credible set of shift configurations comprises the set of distinct, sampled configurations that sum to $95 \%$ of the posterior probability. In the ML tree, all of the configurations within this credible set contain shifts either at the base of Stenodermatinae or at an ancestral noctilionoid node. The different shift configurations in the credible set allow us to quantify uncertainty in placement of a stenodermatine shift or a more broadly encompassing shift. The shift configuration sampled at the highest frequency, $64 \%$, contains only a stenodermatine shift, with all other configurations sampled at a frequency of $12 \%$ or lower (Fig. S4).

\section{MACROEVOLUTIONARY COHORT ANALYSIS}

We can use our results to calculate the probability of clades being independent macroevolutionary cohorts. Our cohort analysis also supports the decoupling of stenodermatines and the rest of bats (Rabosky et al. 2014a). In our ML phylogeny, we reveal striking homogeneity that unites almost all extant bats in one cohort, except the stenodermatines (Fig. 5A). It is clear that there is essentially a $0 \%$ probability of stenodermatines being part of the same cohort as all non-phyllostomids. There is weaker support for other phyllostomids also being part of a distinct cohort apart from other bats. A distinct stenodermatine cohort appears to be somewhat robust to topological uncertainty, though this pattern becomes more equivocal (Fig. 5B). Interestingly, these bootstrap topology analyses highlight a decoupled Pteropus cohort. It is unclear what drives this pattern, as there is no Pteropus signal in the 
original tree

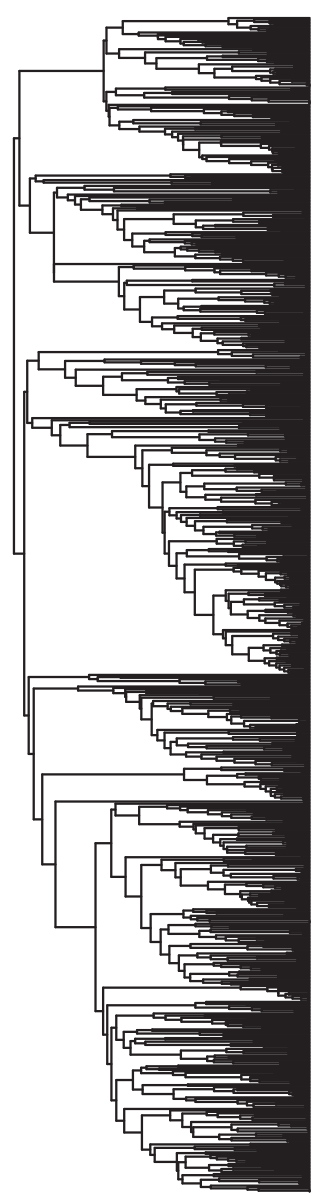

marginal probability

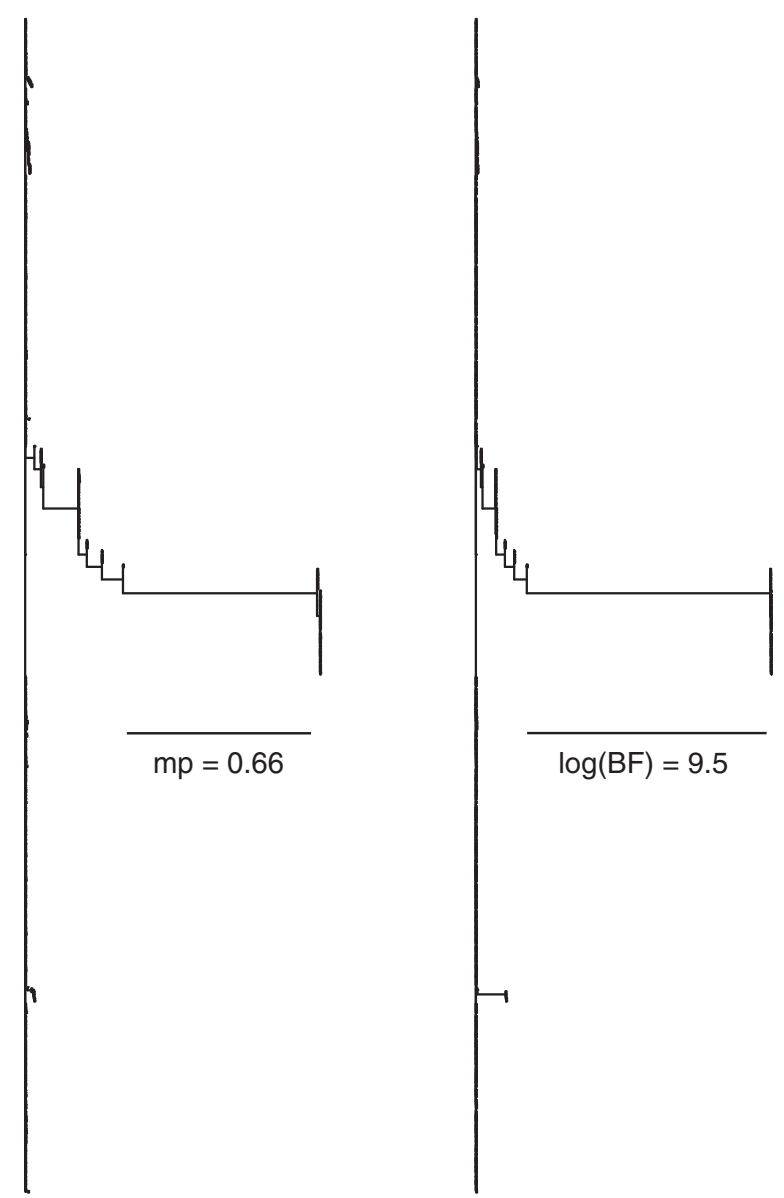

Figure 3. Two different methods of weighing the relative evidence of a regime shift occurring along any individual branch. In the marginal probability (mp) tree, each individual branch length corresponds to the $\mathrm{mp}$ of a shift occurring along that branch across the posterior distribution of BAMM results. In the marginal Bayes factor (BF) tree, each individual branch length represents relative shift density in relation to the prior distribution. The $M L$ tree topology from Figure 2 is included for reference. The longest branch length, in both $\mathrm{mp}$ or $\log (\mathrm{BF})$, is labeled for reference. In both cases, the longest branch is at the base of the subfamily Stenodermatinae.

ML phylogeny, but it is possible this is a product of low genuslevel sampling (Table S2). Our BAMM analysis with all sampling fractions halved, which tests sensitivity to incomplete sampling, is extremely similar with respect to our findings about macroevolutionary homogeneity, diversification rate, and the uniqueness of Stenodermatinae (Fig. S5).

\section{AGE-RICHNESS RELATIONSHIPS}

We explore the effect of age on diversity using age-richness correlations. A Spearman's rank-correlation test for a relationship between crown ages and species richnesses is highly significant, revealing a positive and linear correlation (Spearman's $\rho=0.766$, $P<0.001)$. The PGLS test for a relationship between crown family ages and $\log$ (species richnesses) is also highly significant ( $t=$ 4.677, $P=0.0003$ ), with a clear positive and linear relationship between age and $\log$ (richness) (Fig. S6).

\section{SPECIATION RATES THROUGH TIME}

Our BAMM results for the ML topology are evidence for decreasing speciation rates through time, both across bats as a whole and within the stenodermatine macroevolutionary cohort (Fig. 6). These results are corroborated by the $\gamma$-statistic, which tests for significance of temporal decelerations in the rate of speciation (Pybus and Harvey 2000). The $\gamma$-statistic of our ML phylogeny is $-10.675(P<0.001)$, and the distribution of $\gamma$-statistics across all 100 bootstrap replicates ranges from -12.649 to $-1.982($ mean $=$ -7.840 , median $=-6.572$, with all $P<0.05)$. We infer from negative values that the internal nodes are significantly closer to the root than expected under a pure-birth model (Pybus and Harvey 2000). Our results are unlikely to be explained by incomplete taxon sampling, assuming sampling is not strongly and nonrandomly biased. True bat diversity would need to be near 3000 species (compared to current estimates around 1300) to 


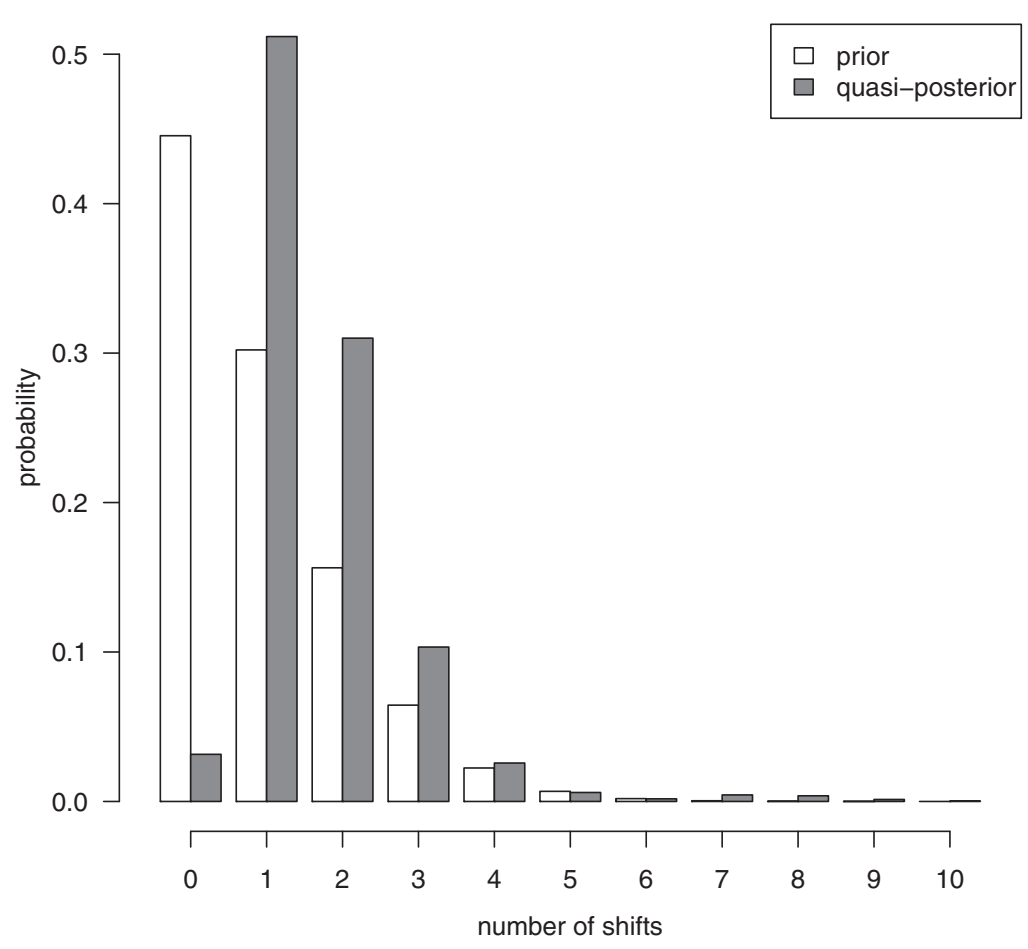

Figure 4. The prior distribution (white) of shifts across the bat phylogeny and the quasi-posterior distribution (gray) of shifts pooled from all the BAMM results on bootstrap replicates. Note that there is nearly zero quasi-posterior probability of zero shifts, and that the highest quasi-posterior probability is for a single shift.

produce an artifactual $\gamma$-statistic as negative as in our ML phylogeny, or would need to be near 2000 to match the bootstraps' mean (Rabosky and Lovette 2008; Table S3).

\section{PHYLOGENETIC IMBALANCE}

Empirical estimates of clade diversity variance, conditioned upon the number of descendants of ancestral lineages at any point in time, are higher than expected under a constant rate birth-death process for the majority of the order's history (Fig. 7). Bats thus appear to have partitioned diversity into clades more unevenly than expected by chance alone, and this pattern has been fairly consistent throughout their history. We only include results between roughly 50 and 15 million years ago. This excludes the early history of the clade, when variances are extremely volatile due to the low number of ancestral lineages, and the last 15 million years, when the effects of incomplete sampling are most dramatic. Results are nearly identical even after excluding the subfamily Stenodermatinae, indicating that this radiation alone does not drive the imbalance (Fig. S7).

\section{Discussion}

\section{BAT DIVERSIFICATION DYNAMICS}

Our macroevolutionary analyses suggest that the global radiation of bats is characterized by a remarkably homogeneous diversifi- cation process. The majority of extant bat species can be united into a single, paraphyletic macroevolutionary cohort (Fig. 5A), with only the subfamily Stenodermatinae having an extremely high probability of being defined by a shift in diversification rate (Fig. 3, Supplementary Fig. 4). Bat speciation dynamics can be characterized by a single global deceleration in the rate of speciation, combined with a recent burst of speciation at the base of the stenodermatines (Fig. 6). We infer stenodermatine diversification rates more than twice that of the rest of the bat radiation, which falls in line with previous studies on this subfamily (Dumont et al. 2012).

Stenodermatinae is a well-studied Neotropical radiation, and diversification rate shifts have previously been inferred at the base of this subfamily. The stenodermatine radiation may be coupled with morphological and behavioral specializations for frugivory (Monteiro and Nogueira 2011; Dumont et al. 2012; Santana et al. 2012). In this framework, stenodermatines are an adaptive radiation nested within the radiation of Chiroptera as a whole, potentially spurred by the key innovation of frugivory (Rojas et al. 2012). Stenodermatine skulls are known to have unique biomechanical properties that can process hard fruit, affording expansion into unexplored niche space and potentially elevating speciation rates (Dumont et al. 2012, 2014).

The relative homogeneity of bat diversification is surprising, especially in the context of past macroevolutionary research. 
A
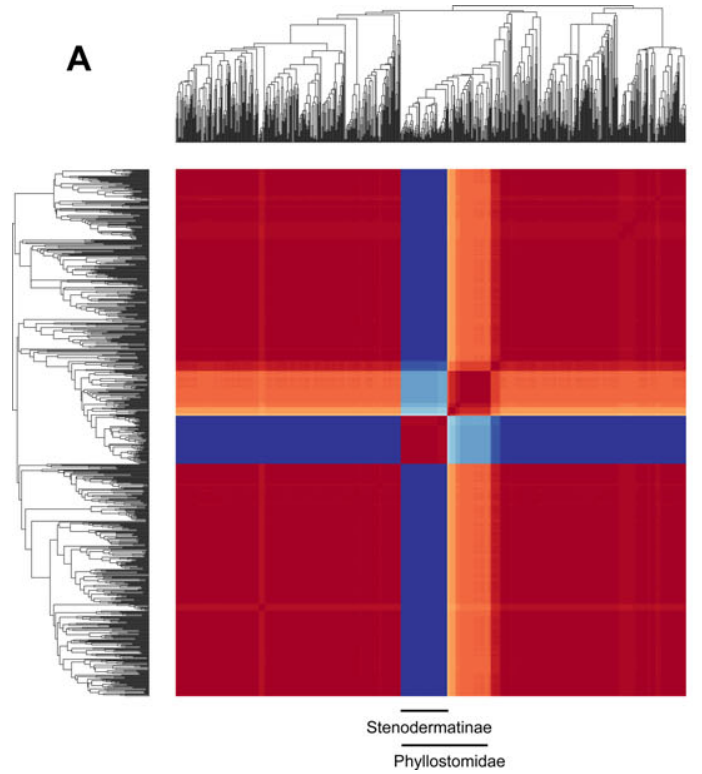

B
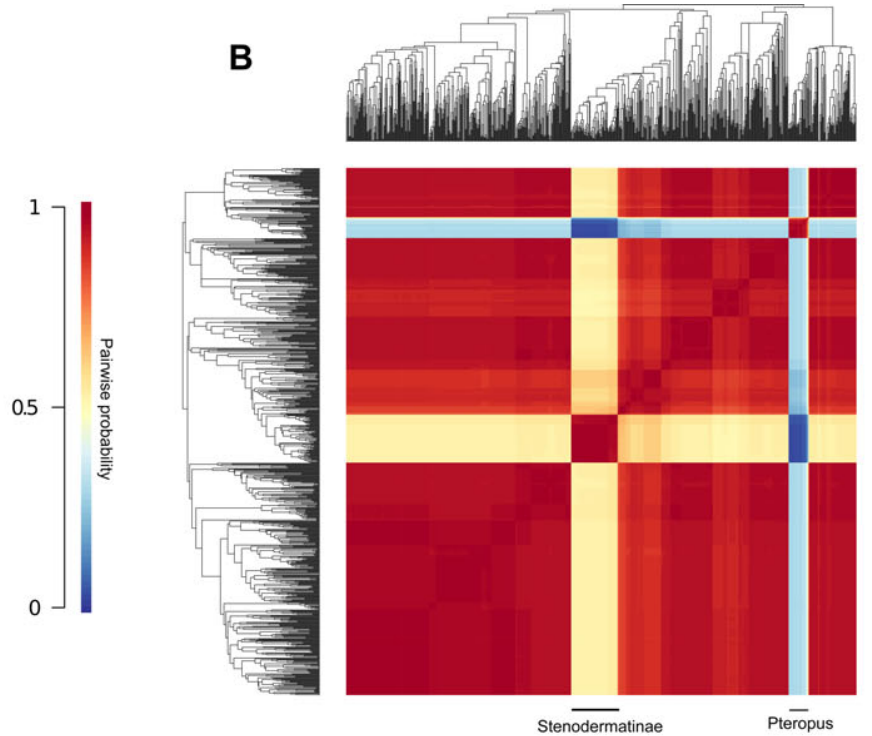

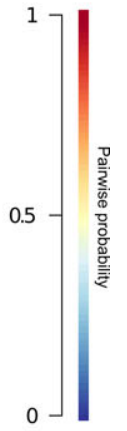

Figure 5. (A) A macroevolutionary cohort matrix for our ML phylogeny of bats. There is only strong evidence for stenodermatines being part of a decoupled diversification regime from other bats. (B) A cohort matrix averaged across all bootstrap replicates to investigate the effect of topological uncertainty. Each point on the main plot represents the average probability across all bootstrap replicates that the two specified branches ever share a macroevolutionary regime. Hence, each individual bootstrap replicate was reshuffled to match the order of branches. We still find evidence for a stenodermatine cohort, but also a Pteropus cohort.

Jones et al. (2005) inferred multiple diversification rate shifts across bats: though the authors found the strongest evidence for rate shifts within Molossidae and Phyllostomidae, they also suggested shifts in the rhinolophoids, vespertilionids, and pteropodids. We do not find strong evidence for this amount of heterogeneity, using a method that explicitly allows for rate heterogeneity and infers probabilities of entire shift configurations. It is unknown how common an overall pattern of relative homogeneity is among other animals. Diversification studies in other metazoans often find considerable rate heterogeneity, perhaps making bats curiously unique given their high diversity (Chan and Moore 2002; Purvis and Agapow 2002; Alfaro et al. 2009; Barker et al. 2013; Rabosky et al. 2013, 2014a). However, previous studies on rate heterogeneity may not be directly comparable to our methods. As the second-most species-rich order of extant mammals, we suggest that bats as a whole may have high diversification rates. This hypothesis can be tested with diversification studies across a time-calibrated, species-level phylogeny of all mammals.

Unresolved or uncertain phylogenetics can compromise accurate macroevolutionary inference. We caution that the phylogeny we present here (Fig. 2, Fig. S8) is not meant to be a systematic reworking of the order, and note some issues that bear future consideration. Finer-scale fossil calibration, especially with methods that explicitly take into account molecular clock variation, can improve our absolute inferences of crown ages. Topologically, groups within Molossidae are not well-supported by our bootstrap replicates. This does not appear to reflect low sequence coverage, as we have molossid data for every included locus. A previous phylogenetic study of Molossidae also raised taxonomic issues regarding genus-level paraphyly that cannot simply be explained by gene tree conflicts (Lamb et al. 2011). Discordances among bootstrap replicates may also reflect incongruence among the concatenated loci, taxonomic uncertainty, extremely rapid divergences, or other evolutionary processes (Knowles 2009; Dávalos et al. 2012; Salichos and Rokas 2013).

Possibly due to these issues, some well-studied genera are paraphyletic in our ML phylogeny. General findings of paraphyly and polyphyly in studies of this scale may be more common than expected (Funk and Omland 2003). Complete resolution of species-level relationships will require careful integration of morphological characters and fossils, as well as more widely available genomic data (Harrison and Kidner 2011; Dávalos et al. 2012; Slater et al. 2012). Even with these caveats, we continue to find signal for a decoupled stenodermatine cohort with respect to phylogenetic uncertainty (Fig. 5B). Because the cohort probabilities are lowered, however, the stenodermatine shift should continue to be revisited as we further resolve bat phylogenetics.

Notably, the flying fox genus Pteropus also appears as a decoupled cohort when accounting for uncertainty, despite being absent from the $95 \%$ credibility set of shift configurations in the ML phylogeny (Fig. S4). This genus, and its family Pteropodidae, may be responsible for the inflated numbers of shifts in the quasi-posterior distribution across all bootstrap topologies, as well 
A

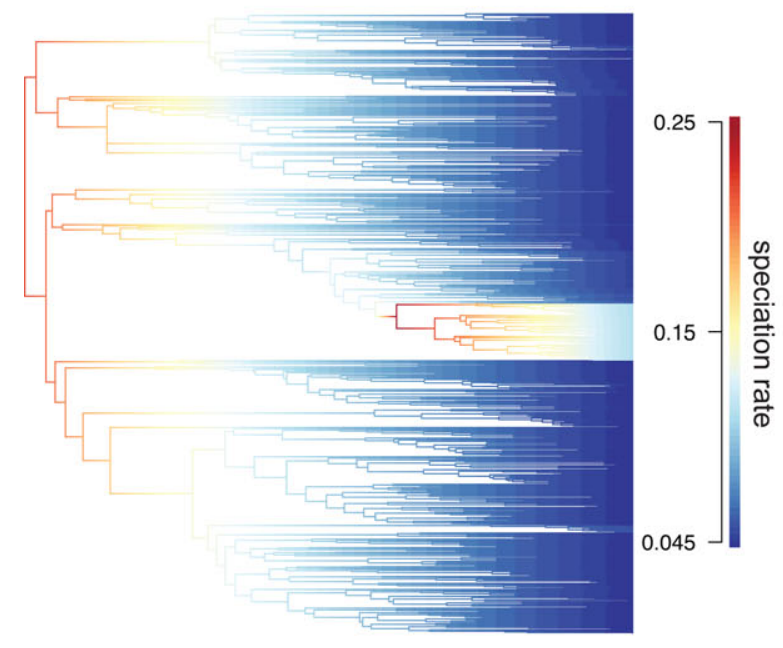

B

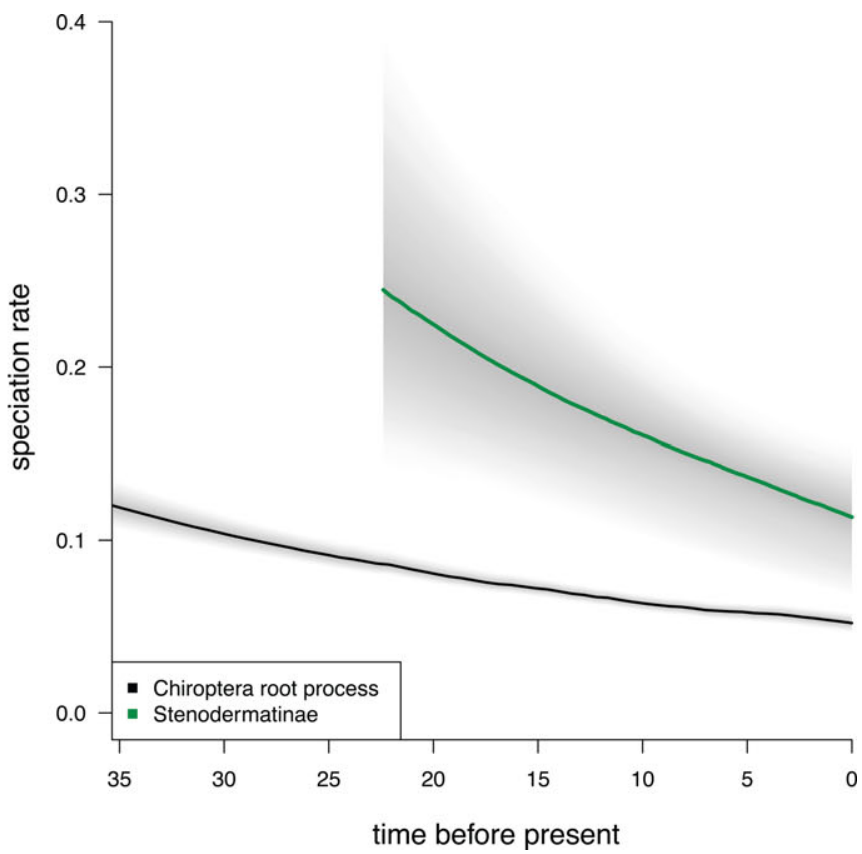

Figure 6. (A) ML phylogeny of bats, with BAMM estimates of instantaneous speciation rate represented by colors along individual branches. (B) Instantaneous speciation rates through time for both stenodermatines (top curve) and nonstenodermatine bats (bottom curve). Around each curve, $90 \%$ credibility intervals from the posterior distribution of BAMM results. Speciation rates are the lambda rate parameters of exponential distributions.

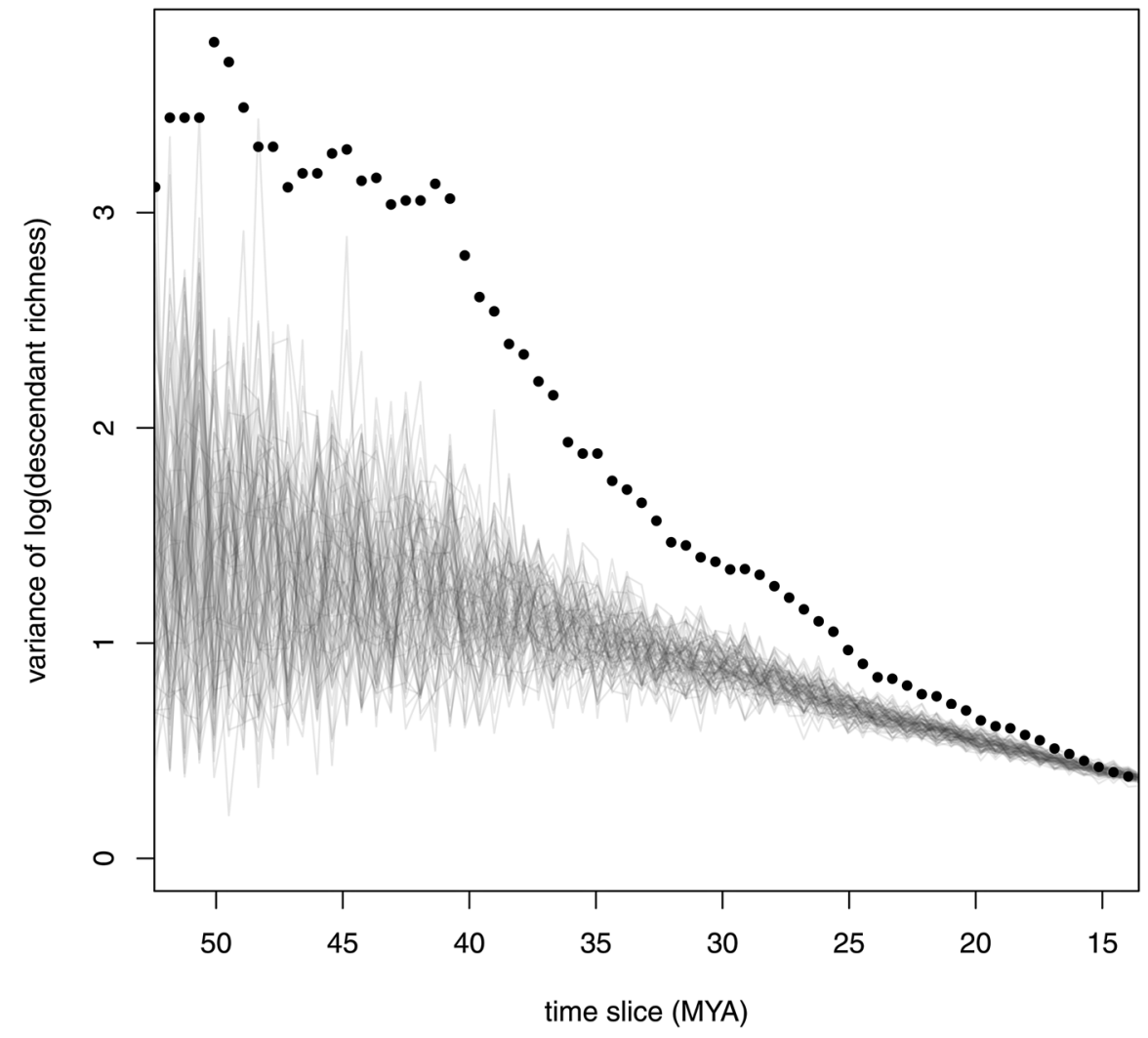

Figure 7. Changes in species richness variance across clades as lineages accumulate through time. Transparent gray lines represent 100 simulations of among-clade variance in species richness as expected under a constant rate birth-death process. Black circles represent empirical variances among clades descended from the lineages that exist at time $t$, in the ML phylogeny of extant bats. For most of bat history, there has been more variance in diversity among bat clades than expected under a constant rate birth-death process. 
(Fig. 4). These Old World fruit bats are known for their speciesrichness and potentially rapid diversification (Almeida et al. 2011), but are also quite undersampled (Table S2). In addition, the family Pteropodidae as a whole is notoriously lacking in fossils (Eiting and Gunnell 2009; Table 2), leading to highly variable node dates (Table 4) across bootstrap replicates and the possibility that this signal is an artifact of both sampling and dating. We suggest that future research pursues the phylogenetics of this group more deeply, with careful analysis of genomic data.

\section{MACROEVOLUTION OF CHIROPTERA}

As we do not find evidence for widespread diversification rate heterogeneity, the possibility remains that patterns of diversity are mostly driven by the effect of clade age (McPeek and Brown 2007). We explore this hypothesis using the crown ages of extant families (Stadler et al. 2014). We find a positive linear relationship between crown family age and richness (Fig. S6), and early family divergences overall. What caused bat families to diverge early in the Eocene, followed by radiations within these families (Fig. 2, Simmons 2005a)? Many large clades are characterized by major ecological and geographic divisions preceding taxonomic, local differentiation (Simpson 1953; Foote 1993; Glor 2010; Sahney et al. 2010; Raia et al. 2012). Declining rates of diversification through time may be one phylogenetic signal of this process: clades switch from early and rapid ecological divergence, to a diversity-dependent slowing of diversification as ecological niches become saturated (Gould et al. 1977; Nee et al. 1992; Rabosky and Lovette 2008; Etienne and Haegeman 2012; Rabosky 2013). We find support for slowdown across the order, with strong evidence for temporal declines in diversification rates throughout bats (Fig. 6) coupled with a significant negative $\gamma$-statistic that is robust to topological uncertainty (Pybus and Harvey 2000).

Specifically, bat diversification appears to be governed by two conflicting macroevolutionary models. The inferred strong slowdown in diversification (Fig. 7) suggests diversity-dependent diversification, yet the significant age-richness relationship (Fig. S6) can be interpreted to contradict this inference (Rabosky 2009a; Wiens 2011). However, diversity-dependence does not preclude increasing diversity through time (Cornell 2013). Support for damped but increasing diversification has been found when investigating other large-scale diversity patterns (Kisel et al. 2011; Cornell 2013). In addition, diversification slowdowns can be caused by other factors, including the mode of speciation or external, abiotic factors (Moen and Morlon 2014), potentially reconciling our findings.

Despite the striking homogeneity of bat diversification, we detect phylogenetic imbalance across bat clades. This imbalance is not explained by the explosive radiation of stenodermatines (Fig. 7, Fig. S7). BAMM may not have enough power to detect certain types of diversification rate heterogeneity that can produce imbalanced phylogenies. In addition, clades at smaller scales (e.g., a single family within one biome) may be more prone to saturation. If these diversity limits are specific to local ecology and biogeography, diversity may be unequally distributed (Rabosky 2009b; Weir and Price 2011). These hypotheses can be tested in the future with phylogenies at the suborder and superfamily scale, where similar diversification regimes may be teased apart, and by explicitly assessing support for damped diversification versus unbounded diversification at different geographic scales.

Many aspects of bat macroevolution remain unknown, but we are beginning to overcome their poor fossil record with widely available genomic data and careful analysis of the available historical data. Van Valen (1979) once mused that, "one may hypothesize that bats did originate, but it is harder to go beyond this." Not only have we moved quite far beyond this initial hypothesis, but our results suggest that bat evolution may be simultaneously more simple and puzzling that even Van Valen could have predicted.

\section{ACKNOWLEDGMENTS}

The authors thank C. Badgley and E. R. Dumont for general research advice, S. A. Smith and C. E. Hinchcliff for computational and methodological assistance, P. O. Title and M. C. Grundler for input on graphics, and two anonymous reviewers for their comments. They also thank C. $\mathrm{J}$. Anderson for developing the MC3 implementation of BAMM, and J. M. Brown for suggesting the use of branch-specific Bayes factors. J. J. S. thanks K. M. Bakker, L. E. Decker, P. R. Glaum, A. L. Gould, M. E. Martinez-Bakker, C. J. H. Miller, and O. Whent for their invaluable support. This research was supported in part by national science foundation (NSF) DEB-1256330. The authors declare no conflicts of interest.

\section{DATA ARCHIVING}

These are currently available on TreeBASE and Dryad.

\section{LITERATURE CITED}

Aberer, A. J., D. Krompass, and A. Stamatakis. 2013. Pruning rogue taxa improves phylogenetic accuracy: an efficient algorithm and webservice. Syst. Biol. 62:162-166.

Agnarsson, I., C. M. Zambrana-Torrelio, N. P. Flores-Saldana, and L. J. May-Collado. 2011. A time-calibrated species-level phylogeny of bats (Chiroptera, Mammalia). PLoS Curr. 3. doi:10.1371/currents.RRN1212.

Alfaro, M. E., F. Santini, C. Brock, H. Alamillo, A. Dornburg, D. L. Rabosky, G. Carnevale, and L. J. Harmon. 2009. Nine exceptional radiations plus high turnover explain species diversity in jawed vertebrates. Proc. Natl. Acad. Sci. USA 106:13410-13414.

Almeida, F. C., N. P. Giannini, R. DeSalle, and N. B. Simmons. 2011. Evolutionary relationships of the old world fruit bats (Chiroptera, Pteropodidae): another star phylogeny? BMC Evol. Biol. 11:281.

Altekar, G., S. Dwarkadas, J. P. Huelsenbeck, and F. Ronquist. 2004. Parallel metropolis coupled Markov chain Monte Carlo for Bayesian phylogenetic inference. Bioinformatics 20:407-415.

Badgley, C., and J. A. Finarelli. 2013. Diversity dynamics of mammals in relation to tectonic and climatic history: comparison of three Neogene records from North America. Paleobiology 39:373-399. 
Barker, F. K., K. J. Burns, J. Klicka, S. M. Lanyon, and I. J. Lovette. 2013. Going to extremes: contrasting rates of diversification in a recent radiation of new world passerine birds. Syst. Biol. 62:298-320.

Barnosky, A. D. 2001. Distinguishing the effects of the Red Queen and Court Jester on Miocene mammal evolution in the northern Rocky Mountains. J. Vertebr. Paleontol. 21:172-185.

Barraclough, T. G., and S. Nee. 2001. Phylogenetics and speciation. Trends Ecol. Evol. 16:391-399.

Benton, M. J. 1987. Progress and competition in macroevolution. Biol. Rev. 62:305-338.

- 2009. The Red Queen and the Court Jester: species diversity and the role of biotic and abiotic factors through time. Science 323:728732 .

Bininda-Emonds, O. R. P., M. Cardillo, K. E. Jones, R. D. E. MacPhee, R. M. D. Beck, R. Grenyer, S. A. Price, R. A. Vos, J. L. Gittleman, and A. Purvis. 2007. The delayed rise of present-day mammals. Nature 446:507-512.

Castresana, J. 2000. Selection of conserved blocks from multiple alignments for their use in phylogenetic analysis. Mol. Biol. Evol. 17:540-552.

Chan, K. M. A., and B. R. Moore. 2002. Whole-tree methods for detecting differential diversification rates. Syst. Biol. 51:855-865.

Cornell, H. V. 2013. Is regional species diversity bounded or unbounded? Biol. Rev. 88:140-165.

Dávalos, L. M., A. L. Cirranello, J. H. Geisler, and N. B. Simmons. 2012. Understanding phylogenetic incongruence: lessons from phyllostomid bats. Biol. Rev. 87:991-1024.

Dumont, E. R., L. M. Dávalos, A. Goldberg, S. E. Santana, K. Rex, and C. C. Voigt. 2012. Morphological innovation, diversification and invasion of a new adaptive zone. Proc. R. Soc. B Biol. Sci. 279:1797-1805.

Dumont, E. R., K. Samadevam, I. Grosse, O. M. Warsi, B. Baird, and L. M. Dávalos. 2014. Selection for mechanical advantage underlies multiple cranial optima in New World leaf-nosed bats. Evolution 68:14361449.

Eiting, T. P., and G. F. Gunnell. 2009. Global completeness of the bat fossil record. J. Mamm. Evol. 16:151-173.

Erwin, D. H., J. W. Valentine, and J. J. Sepkoski, Jr. 1987. A comparative study of diversification events: the early Paleozoic versus the Mesozoic. Evolution 41:1177-1186.

Etienne, R. S., and B. Haegeman. 2012. A conceptual and statistical framework for adaptive radiations with a key role for diversity dependence. Am. Nat. 180:E75-E89.

Farrell, B. D. 1998. "Inordinate fondness" explained: why are there so many beetles? Science 281:555-559.

Fenton, M. B. 2010. Convergences in the diversification of bats. Curr. Zool. 56:454-468.

FitzJohn, R. G., W. P. Maddison, and S. P. Otto. 2009. Estimating traitdependent speciation and extinction rates from incompletely resolved phylogenies. Syst. Biol. 58:595-611.

Foote, M. 1993. Contributions of individual taxa to overall morphological disparity. Paleobiology 19:403-419.

Funk, D. J., and K. E. Omland. 2003. Species-level paraphyly and polyphyly: frequency, causes, and consequences, with insights from animal mitochondrial DNA. Annu. Rev. Ecol. Evol. Syst. 34:397-423.

Glor, R. E. 2010. Phylogenetic insights on adaptive radiation. Annu. Rev. Ecol. Evol. Syst. 41:251-270.

Gould, S. J., D. M. Raup, and J. J. Sepkoski, Jr. 1977. The shape of evolution: a comparison of real and random clades. Paleobiology 3:23-40.

Gould, S. J., N. L. Gilinsky, and R. Z. German. 1987. Asymmetry of lineages and the direction of evolutionary time. Science 236:1437-1441.

Harmon, L. J. 2012. An inordinate fondness for eukaryotic diversity. PLoS Biol. 10:e1001382.
Harmon, L. J., J. T. Weir, C. D. Brock, R. E. Glor, and W. Challenger. 2008. GEIGER: investigating evolutionary radiations. Bioinformatics 24:129131.

Harrison, N., and C. A. Kidner. 2011. Next-generation sequencing and systematics: what can a billion base pairs of DNA sequence data do for you? Taxon 60:1552-1566.

Heath, T. A., D. J. Zwickl, J. Kim, and D. M. Hillis. 2008. Taxon sampling affects inferences of macroevolutionary processes from phylogenetic trees. Syst. Biol. 57:160-166.

Hinchliff, C. E., and E. H. Roalson. 2013. Using supermatrices for phylogenetic inquiry: an example using the sedges. Syst. Biol. 62:205-219.

Jones, K. E., A. Purvis, A. MacLarnon, O. R. P. Bininda-Emonds, and N. B. Simmons. 2002. A phylogenetic supertree of the bats (Mammalia: Chiroptera). Biol. Rev. 77:223-259.

Jones, K. E., O. R. P. Bininda-Emonds, and J. L. Gittleman. 2005. Bats, clocks, and rocks: diversification patterns in Chiroptera. Evolution 59:2243-2255.

Kass, R. E., and A. E. Raftery. 1995. Bayes factors. J. Am. Stat. Assoc. 90:773-795.

Kirkpatrick, M., and M. Slatkin. 1993. Searching for evolutionary patterns in the shape of a phylogenetic tree. Evolution 47:1171-1181.

Kisel, Y., L. McInnes, N. H. Toomey, and C. D. L. Orme. 2011. How diversification rates and diversity limits combine to create large-scale species-area relationships. Philos. Trans. R. Soc. B Biol. Sci. 366:25142525.

Knowles, L. L. 2009. Estimating species trees: methods of phylogenetic analysis when there is incongruence across genes. Syst. Biol. 58:463-467.

Lack, J. B., and R. A. Van Den Bussche. 2010. Identifying the confounding factors in resolving phylogenetic relationships in Vespertilionidae. J. Mammal. 91:1435-1448.

Lamb, J. M., T. M. C. Ralph, T. Naidoo, P. J. Taylor, F. Ratrimomanarivo, W. T. Stanley, and S. M. Goodman. 2011. Toward a molecular phylogeny for the Molossidae (Chiroptera) of the Afro-Malagasy region. Acta Chiropterol. 13:1-16.

Magallón, S., and M. J. Sanderson. 2001. Absolute diversification rates in angiosperm clades. Evolution 55:1762-1780.

Martins, E. P., and T. F. Hansen. 1997. Phylogenies and the comparative method: a general approach to incorporating phylogenetic information into the analysis of interspecific data. Am. Nat. 149:646-667.

McPeek, M. A., and J. M. Brown. 2007. Clade age and not diversification rate explains species richness among animal taxa. Am. Nat. 169:E97-E106.

Meredith, R. W., J. E. Janečka, J. Gatesy, O. A. Ryder, C. A. Fisher, E. C. Teeling, A. Goodbla, E. Eizirik, T. L. L. Simão, T. Stadler, et al. 2011. Impacts of the Cretaceous terrestrial revolution and KPg extinction on mammal diversification. Science 334:521-524.

Miller-Butterworth, C. M., W. J. Murphy, S. J. O’Brien, D. S. Jacobs, M. S. Springer, and E. C. Teeling. 2007. A family matter: conclusive resolution of the taxonomic position of the long-fingered bats, Miniopterus. Mol. Biol. Evol. 24:1553-1561.

Moen, D., and H. Morlon. 2014. Why does diversification slow down? Trends Ecol. Evol. 29:190-197.

Monteiro, L. R., and M. R. Nogueira. 2011. Evolutionary patterns and processes in the radiation of phyllostomid bats. BMC Evol. Biol. 11:137.

Nee, S., A. O. Mooers, and P. H. Harvey. 1992. Tempo and mode of evolution revealed from molecular phylogenies. Proc. Natl. Acad. Sci. USA 89:8322-8326.

Nee, S., E. C. Holmes, R. M. May, and P. H. Harvey. 1994. Extinction rates can be estimated from molecular phylogenies. Philos. Trans. R. Soc. B Biol. Sci. 344:77-82.

Nowak, M. D. 1994. Walker's bats of the world. Johns Hopkins University Press, Baltimore. 
Platt II, R. N., M. W. Vandewege, C. Kern, C. J. Schmidt, F. G. Hoffmann, and D. A. Ray. 2014. Large numbers of novel miRNAs originate from DNA transposons and are coincident with a large species radiation in bats. Mol. Biol. Evol. 31:1536-1545.

Purvis, A., and P. M. Agapow. 2002. Phylogeny imbalance: taxonomic level matters. Syst. Biol. 51:844-854.

Pybus, O. G., and P. H. Harvey. 2000. Testing macro-evolutionary models using incomplete molecular phylogenies. Proc. R. Soc. B Biol. Sci. 267:2267-2272.

Rabosky, D. L. 2009a. Ecological limits and diversification rate: alternative paradigms to explain the variation in species richness among clades and regions. Ecol. Lett. 12:735-743.

2009b. Ecological limits on clade diversification in higher taxa. Am. Nat. 173:662-674.

- 2010. Extinction rates should not be estimated from molecular phylogenies. Evolution 64:1816-1824.

- 2013. Diversity-dependence, ecological speciation, and the role of competition in macroevolution. Annu. Rev. Ecol. Evol. Syst. 44:481502 .

- 2014. Automatic detection of key innovations, rate shifts, and diversity-dependence on phylogenetic trees. PLoS One 9:e89543.

Rabosky, D. L., and I. J. Lovette. 2008. Explosive evolutionary radiations: decreasing speciation or increasing extinction through time? Evolution 62:1866-1875.

Rabosky, D. L., F. Santini, J. Eastman, S. A. Smith, B. Sidlauskas, J. Chang, and M. E. Alfaro. 2013. Rates of speciation and morphological evolution are correlated across the largest vertebrate radiation. Nat. Commun. 4:1958.

Rabosky, D. L., S. C. Donellan, M. C. Grundler, and I. J. Lovette. 2014a. Analysis and visualization of complex macroevolutionary dynamics: an example from Australian scincid lizards. Syst. Biol. 63:610627.

Rabosky, D. L., M. C. Grundler, C. J. R. Anderson, P. O. Title, J. J. Shi, J. W. Brown, H. Huang, and J. G. Larson. 2014b. BAMMtools: an R package for the analysis of evolutionary dynamics on phylogenetic trees. Methods Ecol. Evol. 5:701-707.

Raia, P., F. Carotenuto, F. Passaro, P. Piras, D. Fulgione, L. Werdelin, J. Saarinen, and M. Fortelius. 2012. Rapid action in the Palaeogene, the relationship between phenotypic and taxonomic diversification in Coenozoic mammals. Proc. R. Soc. B Biol. Sci. 280:20122244.

Raup, D. M., S. J. Gould, T. J. M. Schopf, and D. S. Simberloff. 1973. Stochastic models of phylogeny and the evolution of diversity. J. Geol. $81: 525-542$

Revell, L. J. 2012. Phytools: an R package for phylogenetic comparative biology (and other things). Methods Ecol. Evol. 3:217-223.

Rojas, D., Á. Vale, V. Ferrero, and L. Navarro. 2012. The role of frugivory in the diversification of bats in the Neotropics. J. Biogeogr. 39:19481960.

Rosenzweig, M. L., and R. D. McCord. 1991. Incumbent replacement: evidence for long-term evolutionary progress. Paleobiology 17:202-213.

Sahney, S., M. J. Benton, and P. A. Ferry. 2010. Links between global taxonomic diversity, ecological diversity and the expansion of vertebrates on land. Biol. Lett. 6:544-547.

Salichos, L., and A. Rokas. 2013. Inferring ancient divergences requires genes with strong phylogenetic signals. Nature 497:327-331.

Sanderson, M. J. 2002. Estimating absolute rates of molecular evolution and divergence times: a penalized likelihood approach. Mol. Biol. Evol. 19:101-109.

Sanderson, M. J., D. Boss, D. Chen, K. A. Cranston, and A. Wehe. 2008. The PhyLoTA browser: processing GenBank for molecular phylogenetics research. Syst. Biol. 57:335-346.
Santana, S. E., I. R. Grosse, and E. R. Dumont. 2012. Dietary hardness, loading behavior, and the evolution of skull form in bats. Evolution 66:2587-2598.

Schluter, D. 2000. The ecology of adaptive radiation. Oxford Univ. Press, Oxford, U.K.

Simmons, N. B. 2005a. An Eocene big bang for bats. Science 307:527-528.

- 2005b. Order Chiroptera. Pp. 312-529 in D. E. Wilson and D. M. Reeder, eds. Mammal species of the world: a taxonomic and geographic reference. Johns Hopkins Univ. Press, Baltimore, MD.

Simmons, N. B., and T. M. Conway. 2003. Evolution of ecological diversity in bats. Pp. 493-535 in T. H. Kunz and M. B. Fenton, eds. Bat ecology. Univ. of Chicago Press, Chicago, IL.

Simmons, N. B., K. L. Seymour, J. Habersetzer, and G. F. Gunnell. 2008 Primitive early Eocene bat from Wyoming and the evolution of flight and echolocation. Nature 451:818-821.

Simpson, G. G. 1953. The major features of evolution. Columbia City Press, New York.

Slater, G. J., L. J. Harmon, D. Wegmann, P. Joyce, L. J. Revell, and M. E. Alfaro. 2012. Fitting models of continuous trait evolution to incompletely sampled comparative data using approximate Bayesian computation. Evolution 66:752-762.

Smith, S. A., and C. W. Dunn. 2008. Phyutility: a phyloinformatics tool for trees, alignments and molecular data. Bioinformatics 24:715-716.

Smith, S. A., and B. C. O'Meara. 2012. treePL: divergence time estimation using penalized likelihood for large phylogenies. Bioinformatics 28:2689-2690.

Smith, S. A., J. M. Beaulieu, and M. J. Donoghue. 2009. Mega-phylogeny approach for comparative biology: an alternative to supertree and supermatrix approaches. BMC Evol. Biol. 9:37.

Springer, M. S., W. J. Murphy, E. Eizirik, and S. J. O'Brien. 2003. Placental mammal diversification and the Cretaceous-Tertiary boundary. Proc. Natl. Acad. Sci. USA 100:1056-1061.

Stadler, T., D. L. Rabosky, R. E. Ricklefs, and F. Bokma. 2014. On age and species richness of higher taxa. Am. Nat. 184:447-455.

Stamatakis, A. 2014. RAxML version 8: a tool for phylogenetic analysis and post-analysis of large phylogenies. Bioinformatics 30:1312-1313.

Stanley, S. M. 1979. Macroevolution: pattern and process. W.H. Freeman, San Francisco, CA.

Strathmann, R. R., and M. Slatkin. 1983. The improbability of animal phyla with few species. Paleobiology 9:97-106.

Talavera, G., and J. Castresana. 2007. Improvement of phylogenies after removing divergent and ambiguously aligned blocks from protein sequence alignments. Syst. Biol. 56:564-577.

Teeling, E. C., O. Madsen, R. A. Van Den Bussche, W. W. de Jong, M. J. Stanhope, and M. S. Springer. 2002. Microbat paraphyly and the convergent evolution of a key innovation in Old World rhinolophoid microbats. Proc. Natl. Acad. Sci. USA 99:1431-1436.

Teeling, E. C., O. Madsen, W. J. Murphy, M. S. Springer, and S. J. O'Brien. 2003. Nuclear gene sequences confirm an ancient link between New Zealand's short-tailed bat and South American noctilionoid bats. Mol. Phylogenet. Evol. 28:308-319.

Teeling, E. C., M. S. Springer, O. Madsen, P. J. J. Bates, S. J. O’Brien, and W. J. Murphy. 2005. A molecular phylogeny for bats illuminates biogeography and the fossil record. Science 307:580-584.

Teeling, E. C., S. Dool, and M. S. Springer. 2012. Phylogenies, fossils, and functional genes: the evolution of echolocation in bats. Pp. 1-22 in G. F. Gunnell and N. B. Simmons, eds. Evolutionary history of bats: fossils, molecules, and morphology. Cambridge Univ. Press, Cambridge, U.K.

Van Valen, L. 1979. The evolution of bats. Evol. Theory 4:103-121.

Vrba, E. S. 1992. Mammals as a key to evolutionary theory. J. Mammal. 73:1-28. 
Weir, J. T., and T. D. Price. 2011. Limits to speciation inferred from times to secondary sympatry and ages of hybridizing species along a latitudinal gradient. Am. Nat. 177:462-469.

Wiens, J. J. 2011. The causes of species richness patterns across space, time, and clades and the role of "ecological limits." Q. Rev. Biol. 86: 75-96.

Yoder, J. B., E. Clancey, S. Des Roches, J. M. Eastman, L. Gentry, W. Godsoe, T. J. Hagey, D. Jochimsen, B. P. Oswald, J. Robertson, et al. 2010. Ecological opportunity and the origin of adaptive radiations. J. Evol. Biol. 23:1581-1596.
Yu, W., Y. Wu, and G. Yang. 2014. Early diversification trend and Asian origin for extent bat lineages. J. Evol. Biol. 27:2204-2218.

Zanne, A. E., D. C. Tank, W. K. Cornwell, J. M. Eastman, S. A. Smith, R. G. FitzJohn, D. J. McGlinn, B. C. O'Meara, A. T. Moles, P. B. Reich, et al. 2014. Three keys to the radiation of angiosperms into freezing environments. Nature 506:89-92.

Associate Editor: M. Alfaro Handling Editor: T. Lenormand

\section{Supporting Information}

Additional Supporting Information may be found in the online version of this article at the publisher's website:

Fossil Calibration (Table 2) References

Supplementary Figure 1: An example cohort matrix constructed using a simulated phylogeny of four taxa, separated into two clades A and B.

Supplementary Figure 2: The null expectation of a constant rate birth-death process for our operational definition of imbalance and its change through time.

Supplementary Figure 3: Support for the ML phylogenetic estimate of 812 extant species of bats based on 29 genetic loci (Table 1). Relevant nodes are labeled in black (70\% support) or grey (50\% support).

Supplementary Figure 4: A set of shift configurations comprising the for most credible shift configurations from our BAMM analysis on the ML phylogeny.

Supplementary Figure 5: A cohort matrix (see Fig. 5, Fig. S1) for the variant of our BAMM analysis with halved sampling fractions.

Supplementary Figure 6: A plot depicting the relationship between crown family age, in millions of years, and $\log$ (richness) for each nonmonotypic family.

Supplementary Figure 7: An analysis of phylogenetic imbalance through time, without the exceptional subfamily Stenodermatinae.

Supplementary Figure 8: A high-resolution version of the ML, time-calibrated phylogeny (Fig. 2) used for macroevolutionary analyses.

Supplementary Table 1: List of taxa removed from the dataset following an initial RAxML search.

Supplementary Table 2: Approximate supermatrix sampling percentages (i.e., at least one locus was included per species) for all extant families of bats; these percentages were used to parameterize BAMM analysis and account for incomplete sampling.

Supplementary Table 3: Phylogenies simulated under a pure birth model with differing levels of incomplete sampling, and the resulting $\gamma$-statistic (Pybus and Harvey 2000) for each level of sampling. 